\title{
Génération mécanique des émulsions
}

\author{
C. Dalmazzone ${ }^{1}$ \\ 1 Institut français du pétrole, 1 et 4, avenue de Bois-Préau, 92852 Rueil-Malmaison Cedex - France \\ e-mail : christine.dalmazzone@ifp.fr
}

\begin{abstract}
Résumé - Les émulsions sont des systèmes complexes très fréquemment rencontrés dans notre vie quotidienne et dans la plupart des secteurs industriels, dont l'industrie pétrolière. La compréhension des mécanismes de formation des émulsions est essentielle pour assurer la maîtrise de leur fabrication ou de leur traitement. Cet article fait une synthèse des principaux phénomènes qui entrent en jeu lors de la formation d'une émulsion. La rupture des gouttelettes en écoulement laminaire ou turbulent est discutée et des corrélations permettant de prédire le diamètre des gouttelettes formées sont données. On présente également le rôle des tensioactifs ainsi que les échelles de temps caractéristiques des différents processus impliqués. Le cas des émulsions pétrolières obtenues en production est étudié plus particulièrement.

Mots-clés: émulsions, formation, émulsification, taille de gouttes, déformation, rupture, cisaillement, turbulence, émulsions pétrolières.
\end{abstract}

\begin{abstract}
Mechanical Formation of Emulsions - Emulsions are complicated systems which are very usually encountered in our everyday life, as well as in most industries, including the petroleum industry. Comprehension of the mechanisms of emulsion formation is essential in order to control their making or their treatment. This paper is a synthesis of the main phenomena that occur during the formation of an emulsion. Droplet break-up in laminar and turbulent flow is discussed and some correlations are given, that allow the prediction of the droplet diameter. The role of surfactants is also presented, as well as the characteristic time scales of the different processes occurring. The case of petroleum emulsions obtained during oil production is particularly studied.
\end{abstract}

Keywords: emulsions, formation, emulsification, droplet size, deformation, breaking, shear, turbulence, crude oil emulsions. 


\section{NOMENCLATURE}

$D$

$D_{c} \quad$ diamètre de la conduite $(\mathrm{m})$

$D_{0}^{c}$ diamètre de l'orifice $(\mathrm{m})$

$d$ diamètre d'une goutte $(\mathrm{m})$

$d_{32}$ diamètre de Sauter $(\mathrm{m})$

$d_{95}$ diamètre maximal pour $95 \%$ de la population de gouttes en volume (m)

$E \quad$ module de dilatation de surface $\left(\mathrm{N} \cdot \mathrm{m}^{-1}=\mathrm{kg} \cdot \mathrm{s}^{-2}\right)$

$E_{f} \quad$ élasticité de Gibbs $\left(\mathrm{N} \cdot \mathrm{m}^{-1}=\mathrm{kg} \cdot \mathrm{s}^{-2}\right)$

$E_{v} \quad$ densité d'énergie $\left(\mathrm{J} \cdot \mathrm{m}^{-3}=\mathrm{kg} \cdot \mathrm{m}^{-1} \cdot \mathrm{s}^{-2}\right)$

$F$ fonction

$F_{d} \quad$ force de déformation $\left(\mathrm{N}=\mathrm{kg} \cdot \mathrm{m} \cdot \mathrm{s}^{-2}\right)$

$\mathrm{g} \quad$ accélération de la gravité $\left(\mathrm{m} \cdot \mathrm{s}^{-2}\right)$

$G \quad$ gradient de vitesse $\left(\mathrm{s}^{-1}\right)$

$\Delta G_{f}$ énergie libre de formation $\left(\mathrm{N} \cdot \mathrm{m}=\mathrm{kg} \cdot \mathrm{m}^{2} \cdot \mathrm{s}^{-2}\right)$

$h \quad$ épaisseur du film $(\mathrm{m})$

$k \quad$ nombre d'onde $=2 \pi / \lambda\left(\mathrm{m}^{-1}\right)$

$K \quad$ constante de vitesse $\left(\mathrm{s}^{-1}\right.$ ou m $\left.\mathrm{m}^{-3} \cdot \mathrm{s}^{-1}\right)$

$L \quad$ plus grande dimension d'une goutte déformée (m)

$m$ concentration molaire $\left(\mathrm{mol} \cdot \mathrm{m}^{-3}\right)$

$N \quad$ nombre par unité de volume $\left(\mathrm{m}^{-3}\right)$

$\Delta P \quad$ différence de pression de Laplace $\left(\mathrm{Pa}=\mathrm{kg} \cdot \mathrm{m}^{-1} \cdot \mathrm{s}^{-2}\right)$

$p \quad$ pression $\left(\mathrm{Pa}=\mathrm{kg} \cdot \mathrm{m}^{-1} \cdot \mathrm{s}^{-2}\right)$

$q$ rapport des viscosités $\eta_{d} / \eta_{c}$

$R \quad$ rayon de courbure (m)

$r \quad$ rayon $(\mathrm{m})$

$r_{\text {cr }} \quad$ rayon critique

$\Delta S_{c} \quad$ variation d'entropie $\left(\mathrm{N} \cdot \mathrm{m} \cdot \mathrm{K}^{-1}=\mathrm{kg} \cdot \mathrm{m}^{2} \cdot \mathrm{s}^{-2} \cdot \mathrm{K}^{-1}\right)$

$t \quad$ temps (s)

$T$ température (K)

$u \quad$ vitesse $\left(\mathrm{m} \cdot \mathrm{s}^{-1}\right)$

$\Delta u \quad$ vitesse de tourbillon $\left(\mathrm{m} \cdot \mathrm{s}^{-1}\right)$

$X \quad$ diamètre de l'agitateur $(\mathrm{m})$

$x \quad$ taille du tourbillon (m)

$\alpha \quad$ amplitude de déformation (m)

$\beta \quad$ facteur de croissance d'instabilité $\left(\mathrm{s}^{-1}\right)$ ou rapport $D_{o} / D_{c}$

$\varepsilon \quad$ densité de puissance $\left(\mathrm{W} \cdot \mathrm{m}^{-3}=\mathrm{kg} \cdot \mathrm{m}^{-1} \cdot \mathrm{s}^{-3}\right)$

$\phi \quad$ fraction volumique de la phase dispersée

$\gamma \quad$ tension interfaciale $\left(\mathrm{N} \cdot \mathrm{m}^{-1}=\mathrm{kg} \cdot \mathrm{s}^{-2}\right)$

$\Gamma \quad$ excès de surface $\left(\mathrm{mol} \cdot \mathrm{m}^{-2} \mathrm{ou} \mathrm{kg} \cdot \mathrm{m}^{-2}\right)$

$\eta \quad$ viscosité $\left(\mathrm{Pa} \cdot \mathrm{s}=\mathrm{kg} \cdot \mathrm{m}^{-1} \cdot \mathrm{s}^{-1}\right)$

$\eta_{c} \quad$ viscosité de la phase continue $\left(\mathrm{Pa} \cdot \mathrm{s}=\mathrm{kg} \cdot \mathrm{m}^{-1} \cdot \mathrm{s}^{-1}\right.$ )

$\eta_{d} \quad$ viscosité de la phase dispersée $\left(\mathrm{Pa} \cdot \mathrm{s}=\mathrm{kg} \mathrm{m}^{-1} \mathrm{~s}^{-1}\right)$

$\lambda$ longueur d'onde (m)

$v \quad$ fréquence $(\mathrm{Hz})$ ou vitesse de révolution $\left(\mathrm{s}^{-1}\right)$ $\theta \quad$ rayon d'une conduite (m)

$\rho \quad$ masse volumique $\left(\mathrm{kg} \cdot \mathrm{m}^{-3}\right)$

$\rho_{c} \quad$ masse volumique de la phase continue $\left(\mathrm{kg} \cdot \mathrm{m}^{-3}\right)$

$\rho_{d} \quad$ masse volumique de la phase dispersée $\left(\mathrm{kg} \cdot \mathrm{m}^{-3}\right)$

$\tau \quad$ temps caractéristique (s)

$\tau_{\text {déf }} \quad$ temps caractéristique de déformation ( $\mathrm{s}$ )

$\tau_{\text {ads }}$ temps caractéristique d'adsorption (s)

$\tau_{\text {col }}$ temps caractéristique de collision (s)

$\tau_{\text {rup }}$ temps caractéristique de rupture (s)

$\tau_{\text {mem }}$ temps caractéristique de relaxation (s)

$\omega$ pulsation $\left(\mathrm{s}^{-1}\right)$

\section{NOMBRES ADIMENSIONNELS}

$\mathrm{Ca}$ nombre capillaire

$\mathrm{Re}$ nombre de Reynolds

$\mathrm{Re}_{g}$ nombre de Reynolds de la goutte

We nombre de Weber

Oh nombre d'Ohnsorge

\section{INTRODUCTION}

Les émulsions font sans aucun doute partie des systèmes complexes les moins connus, bien que côtoyées par tout un chacun dans tous les aspects de la vie quotidienne. Elles se rencontrent dans le domaine alimentaire (lait, mayonnaise, etc.), les cosmétiques (crèmes et lotions), la pharmacie (crèmes, dérivés vitaminés ou hormonaux, etc.) et dans un grand nombre de produits liés à l'activité agricole (insecticides ou herbicides). Ces émulsions peuvent être des produits naturels ou formulés, recherchés pour des propriétés particulières, ou à l'inverse, des systèmes non souhaités qui apparaissent dans le cadre d'un procédé industriel.

Les émulsions pétrolières, qui ne sont certes pas aussi familières, se rencontrent cependant à tous les niveaux de l'industrie pétrolière (Schramm, 1992): fluides de forage, production, raffineries et transport. Dans cette industrie coexistent par ailleurs des émulsions désirables et indésirables. Les émulsions que l'on subit sont les émulsions formées en tête de puits, les émulsions qui résultent de procédés de flottation, les émulsions formées en mer après un déversement par exemple. Les émulsions désirées sont celles qui résultent de la mise en émulsion des bruts lourds, les fluides de forage, les bitumes ou celles qui proviennent de la récupération assistée.

Cet article de synthèse a pour but de faire un bilan le plus complet possible sur l'état des connaissances en matière de génération mécanique des émulsions. L'objectif est de présenter les mécanismes majeurs qui régissent la formation des émulsions. Les principaux points abordés sont les aspects thermodynamiques et énergétiques, les différents processus impliqués dans la mise en émulsion, les échelles de temps 
mises en jeu, les différentes techniques d'émulsification et les mécanismes de formation du film interfacial et des gouttelettes, sans perdre de vue l'impact de la coalescence sur le processus global d'émulsification. En dernier lieu, et à titre d'illustration du problème concret rencontré dans le domaine pétrolier, le cas particulier des émulsions pétrolières obtenues en tête de puits est traité.

\section{ASPECTS THERMODYNAMIQUES ET ÉNERGÉTIQUES}

En général, deux méthodes sont utilisées pour préparer des dispersions: on peut fabriquer des particules à partir d'unités moléculaires (nucléation et croissance) ou subdiviser un matériau en petites unités (broyage ou émulsification). Le processus d'émulsification, qui concerne au sens large la dispersion d'un liquide dans un autre liquide, est gouverné par les forces interfaciales. L'énergie libre de formation de gouttelettes à partir du liquide $\left(\Delta G_{f}\right)$ s'exprime selon l'équation (1):

$$
\Delta G_{f}=\gamma \Delta A-T \Delta S_{c}
$$

avec $\Delta A$ augmentation de l'aire interfaciale, $\gamma$ tension interfaciale entre les deux liquides et $T \Delta S_{c}$ contribution d'entropie liée à l'augmentation du nombre de gouttelettes. En général, $\gamma \Delta A \gg T \Delta S_{c}$, et par conséquent l'émulsification est un processus non spontané. L'énergie nécessaire à l'émulsification est supérieure de plusieurs ordres de grandeur à l'énergie nécessaire à la création d'une nouvelle surface $(\gamma \Delta A)$. En fait, cette énergie peut s'exprimer selon l'équation de YoungLaplace:

$$
\Delta P=\gamma\left(1 / R_{1}+1 / R_{2}\right)
$$

avec $\Delta P$ différence de pression de Laplace, et $R_{1}$ et $R_{2}$ rayons principaux de courbure. Pour une gouttelette sphérique de rayon $r, \Delta P$ est égale à $2 \gamma / r$. Prenons l'exemple de gouttelettes d'huile de $1 \mu \mathrm{m}$ de rayon formées dans l'eau, avec une fraction volumique $\phi$ de 0,1 et une tension interfaciale de $0,01 \mathrm{~N} / \mathrm{m}$. L'énergie libre de surface n'est que de $3 \mathrm{~kJ} / \mathrm{m}^{3}$, mais l'énergie requise est essentiellement due au gradient de pression de Laplace, de l'ordre de $2 \times 10^{10} \mathrm{~Pa} / \mathrm{m}\left(2 \gamma / r^{2}\right)$, soit un gradient de vitesse de $10^{7} \mathrm{~s}^{-1}$ si l'on considère une viscosité de $1 \mathrm{mPa} \cdot \mathrm{s}$ pour la phase continue. Cette quantité d'énergie ne peut être fournie que par une agitation intense, qui va ensuite être dissipée sous forme de chaleur.

L'intensité de l'agitation est gouvernée en grande partie par la densité de puissance $\varepsilon$ dans le liquide, c'est-à-dire la quantité d'énergie mécanique dissipée par unité de volume et de temps. Par conséquent, l'efficacité de l'émulsification dépend essentiellement de $\varepsilon$. Pour obtenir une mise en émulsion efficace, il faut dissiper l'énergie disponible dans le temps le plus court possible.

L'équation (2) met bien en évidence l'influence des tensioactifs, qui, en abaissant la tension interfaciale $\gamma$, diminuent la quantité d'énergie nécessaire et favorisent ainsi la mise en émulsion. On peut alors réduire l'énergie mécanique nécessaire d'un facteur 10 .

\section{PROCESSUS IMPLIQUÉS DANS L'ÉMULSIFICATION}

D'un point de vue pratique, on fabrique une émulsion en appliquant aux deux liquides concernés une énergie mécanique. L'interface se déforme alors jusqu'à la formation de gouttelettes. Les gouttelettes résultantes sont en général trop grosses et doivent être divisées en très petites gouttelettes: cette division des gouttelettes est une étape critique du processus d'émulsification.

Pour qu'il y ait création d'une nouvelle surface, il faut qu'il y ait déformation de la phase dispersée. Cette déformation s'oppose à la force de Laplace, comme nous l'avons vu précédemment. Plus les gouttelettes sont petites, plus la force de Laplace qui s'oppose à une déformation en gouttes plus petites est élevée.

Les gouttelettes peuvent se déformer sous l'action des forces visqueuses ou d'inertie. La contrainte extérieure appliquée à la goutte doit être du même ordre de grandeur que la pression de Laplace. Les gradients de vitesse ou de pression requis sont en général fournis par l'agitation. Plus l'on souhaite de gouttelettes de faible diamètre, plus l'agitation doit être importante. En général, le régime d'écoulement du liquide pendant l'émulsification est turbulent, sauf si la viscosité de la phase continue est élevée.

Il est difficile de fabriquer des émulsions sans agents tensioactifs. Le tensioactif agit en fait en abaissant la valeur de la tension interfaciale, ce qui conduit à une diminution de la pression de Laplace pour une même taille de gouttes. La présence du tensioactif est également nécessaire à la formation du film interfacial. Son rôle est par conséquent essentiel; pour être actif, il doit être transporté à l'interface où il s'adsorbe et forme une couche interfaciale. Le transport du tensioactif est bien sûr amélioré par l'agitation.

Les gouttelettes peuvent recoalescer aussitôt après leur formation. C'est également une étape critique du processus d'émulsification. À ce niveau, le choix du tensioactif est primordial. Il détermine largement la nature de la phase continue; c'est en général la phase où le tensioactif est soluble (règle de Bancroft). La vitesse de coalescence des gouttelettes de la phase dispersée dépend essentiellement de la nature et de la concentration du tensioactif. Les gouttelettes peuvent floculer pendant l'émulsification, mais les flocs sont en général très rapidement dispersés.

Tous les processus que nous venons de passer en revue ont lieu simultanément, et leur vitesse dépend toujours de plusieurs facteurs. Il faut également garder à l'esprit que chaque processus influence le suivant. L'ensemble de ces remarques met bien en évidence la complexité du processus global d'émulsification. 


\section{3 ÉCHELLES DE TEMPS}

Étant donné le nombre de processus impliqués dans l'émulsification, il apparaît intéressant d'avoir un ordre de grandeur de leurs temps caractéristiques.

On relève trois temps caractéristiques: le temps de déformation $\tau_{\text {déf }}$, le temps d'adsorption $\tau_{\text {ads }}$ et le temps de collision $\tau_{\text {col }}$.

Le temps de relaxation qui suit une petite déformation de la goutte (à tension interfaciale $\gamma$ constante et sans effets inertiels) peut s'exprimer de la façon suivante (Levich, 1962):

$$
\tau_{\text {déf }} \approx \eta_{d} r / \gamma
$$

avec $\eta_{d}$ viscosité de la phase dispersée.

Le temps caractéristique nécessaire au transport du tensioactif vers la nouvelle interface est déterminé essentiellement par la convection, et non par la diffusion, comme on pourrait le penser a priori. En flux laminaire, pour une goutte de rayon $r$ (Levich, 1962):

$$
\tau_{\mathrm{ads}} \approx(3 \pi \Gamma) /\left(r m_{c} G\right) \approx\left(10^{4} r m_{c} G\right)^{-1}
$$

et en régime turbulent isotrope (Levich, 1962; Spielman, 1978):

$$
\tau_{\mathrm{ads}} \approx\left(5 \Gamma \eta_{c}^{0,5}\right) /\left(r m_{c} \varepsilon^{0,5}\right) \approx\left(10^{4} r m_{c}\right)^{-1}\left(\varepsilon / \eta_{c}\right)^{-0,5}
$$

avec $\Gamma$ excès de surface, $\eta_{c}$ viscosité de la phase continue, $m_{c}$ concentration molaire du tensioactif et $\varepsilon$ densité de puissance.

On ne peut pas prédire les vitesses de coalescence. La théorie des collisions (Levich, 1962; Spielman, 1978) nous donne, pour de faibles fractions dispersées, un temps moyen entre les collisions pour une gouttelette. Dans un écoulement laminaire:

$$
\tau_{\mathrm{col}}=\pi /(8 \phi G)
$$

et pour un écoulement turbulent isotrope:

$$
\tau_{\mathrm{col}} \approx\left(r^{2 / 3} \rho_{c}^{1 / 3}\right) /\left(10 \phi \varepsilon^{1 / 3}\right)
$$

avec $\phi$ fraction volumique de la phase dispersée et $G$ gradient de vitesse.

Le tableau 1 reprend des exemples de temps caractéristiques rencontrés dans les différents processus impliqués dans l'émulsification (Walstra, 1983).

\section{MOYENS ET TECHNIQUES DE FABRICATION DES ÉMULSIONS}

De nombreux appareillages ont été conçus pour fabriquer des émulsions (Becher, 1965). Le tableau 2 reprend les principaux systèmes utilisés (Walstra, 1983).

Parmi toutes ces techniques, certaines sont utilisées uniquement au laboratoire (secoueurs, vibreurs, magnétostriction, électricité, aérosols, condensation). Les méthodes les plus impliquées dans les applications industrielles sont les rotor-stator, les moulins à colloïdes, les homogénéisateurs haute pression et les ultrasons. Il faut noter que la plupart du temps, on emploie deux techniques combinées, par exemple injection plus homogénéisateur haute pression ou agitation

\begin{tabular}{|c|c|c|c|c|}
\hline Conditions * & \multicolumn{2}{|c|}{ Écoulement laminaire } & \multicolumn{2}{|c|}{ Écoulement turbulent } \\
\hline$G\left(\mathrm{~s}^{-1}\right)$ & $10^{4}$ & $10^{5}$ & - & - \\
\hline$\varepsilon\left(\mathrm{W} \cdot \mathrm{m}^{-3}\right)$ & - & - & $3 \cdot 10^{7}$ & $10^{10}$ \\
\hline$\eta_{c}(\mathrm{mPa} \cdot \mathrm{s})$ & 100 & 100 & 1 & 1 \\
\hline$\eta_{d}(\mathrm{mPa} \cdot \mathrm{s})$ & 1 & 1 & 100 & 100 \\
\hline$r_{\mathrm{cr}}(\mu \mathrm{m})$ & 10 & 1 & 10 & 1 \\
\hline$\tau_{\text {déf }}(\mathrm{ms})$ pour $r=1 \mathrm{~mm}$ & $(0,1)$ & 0,1 & 10 & 10 \\
\hline$\tau_{\text {déf }}(\mathrm{ms})$ pour $r=r_{\mathrm{cr}}$ & $(0,1)$ & $(0,01)$ & 0,1 & 0,01 \\
\hline$\tau_{\text {ads }}(\mathrm{ms})$ pour $r=1 \mathrm{~mm}$ & $10^{-3}$ & $10^{-4}$ & $0,5 * *$ & $0,05^{* *}$ \\
\hline$\tau_{\mathrm{ads}}(\mathrm{ms})$ pour $r=r_{\mathrm{cr}}$ & 0,1 & 0,1 & 6 & 3 \\
\hline$\tau_{\mathrm{col}}(\mathrm{ms})$ & 0,4 & 0,04 & $0,01 * * *$ & $0,0005^{* * *}$ \\
\hline
\end{tabular}
plus homogénéisateur.

TABLEAU 1

Échelles de temps des processus impliqués dans l'émulsification (d'après Walstra, 1983)

Time scales of processes involved in emulsification (from Walstra, 1983)

* Autres conditions : $\gamma=10 \mathrm{mN} \cdot \mathrm{m}^{-1} ; \quad \rho_{d}=\rho_{c}=10^{3} \mathrm{~kg} \cdot \mathrm{m}^{-3} ; \quad \phi=0,1 ; \quad m=10 \mathrm{~mol} \cdot \mathrm{m}^{-3}$

** Estimation grossière

$* * *$ Pour $r=r_{\text {cr }}$ 
TABLEAU 2

Les différents appareillages utilisés pour fabriquer des émulsions (d'après Walstra, 1983) Different apparatuses used for emulsion making (from Walstra, 1983)

\begin{tabular}{|c|c|c|c|c|c|c|}
\hline Technique & $\begin{array}{c}\text { Technique } \\
\text { comparable }\end{array}$ & $\begin{array}{l}\text { Formation } \\
\text { de gouttes }\end{array}$ & $\begin{array}{l}\text { Mécanisme } \\
\text { principal }\end{array}$ & $\begin{array}{l}\text { Densité } \\
\text { d'énergie }\end{array}$ & $\begin{array}{c}\text { Mode } \\
\text { opératoire }\end{array}$ & $\begin{array}{l}\text { Phase } \\
\text { continue }\end{array}$ \\
\hline 1. Secouage & $4 a$ & + & (turbulence) & faible & batch & peu visqueuse \\
\hline $\begin{array}{l}\text { 2. Écoulement } \\
\text { a. laminaire } \\
\text { b. turbulent }\end{array}$ & $\begin{array}{c}5 \\
4 a\end{array}$ & $\begin{array}{l}(+) \\
+\end{array}$ & $\begin{array}{l}\text { forces visqueuses } \\
\text { turbulence }\end{array}$ & $\begin{array}{l}\text { faible-moyen } \\
\text { faible-moyen }\end{array}$ & $\begin{array}{l}\text { continu } \\
\text { continu }\end{array}$ & $\begin{array}{c}\text { visqueuse } \\
\text { peu visqueuse }\end{array}$ \\
\hline 3. Injection (jet) & $10 \mathrm{a}$ & + & - & faible & continu & - \\
\hline $\begin{array}{l}\text { 4. Agitation } \\
\text { a. simple } \\
\text { b. rotor-stator } \\
\text { c. racleur } \\
\text { d. vibreur }\end{array}$ & $\begin{array}{c}1,2 \mathrm{~b} \\
(5) \\
5 \\
8 \mathrm{a}\end{array}$ & $\begin{array}{l}+ \\
+ \\
+ \\
+\end{array}$ & $\begin{array}{c}\text { turbulence, f. visq. } \\
\text { turbulence, f. visq. } \\
\text { forces visqueuses } \\
\text { ? }\end{array}$ & $\begin{array}{c}\text { faible } \\
\text { moyen-élevé } \\
\text { faible-moyen } \\
\text { faible }\end{array}$ & $\begin{array}{c}\text { batch } \\
\text { ou } \\
\text { continu }\end{array}$ & $\begin{array}{c}\text { visqueuse } \\
\text { peu visqueuse }\end{array}$ \\
\hline 5. Moulin à colloïdes & $2 \mathrm{a}, 4 \mathrm{c}, 6$ & $(+)$ & forces visqueuses & moyen-élevé & continu & visqueuse \\
\hline $\begin{array}{l}\text { 6. Broyeur à billes } \\
\text { et à galets }\end{array}$ & 5 & + & forces visqueuses & moyen & $\begin{array}{c}\text { batch } \\
\text { (continu) }\end{array}$ & visqueuse \\
\hline $\begin{array}{l}\text { 7. Homogénéisateur } \\
\text { haute pression }\end{array}$ & $(2 b)$ & - & $\begin{array}{l}\text { turbulence, cavitation } \\
\text { et forces visqueuses }\end{array}$ & élevé & continu & peu visqueuse \\
\hline $\begin{array}{l}\text { 8. Ultrasons } \\
\text { a. couteau vibrant } \\
\text { b. magnétostriction }\end{array}$ & $4 d$ & $\begin{array}{l}+ \\
+\end{array}$ & $\begin{array}{c}\text { cavitation et turbulence } \\
\text { cavitation }\end{array}$ & $\begin{array}{l}\text { moyen-élevé } \\
\text { moyen-élevé }\end{array}$ & $\begin{array}{l}\text { continu } \\
\text { batch ou continu }\end{array}$ & $\begin{array}{l}\text { aqueuse } \\
\text { aqueuse }\end{array}$ \\
\hline 9. Électrique & $10 \mathrm{~b}$ & - & $\begin{array}{l}\text { charge } \\
\text { électrique }\end{array}$ & moyen & $\begin{array}{c}\text { batch } \\
\text { (continu) }\end{array}$ & $\begin{array}{l}\text { plusieurs } \\
\text { restrictions }\end{array}$ \\
\hline $\begin{array}{l}\text { 10. Aérosols } \\
\text { a. mécanique } \\
\text { b. électrique }\end{array}$ & $\begin{array}{l}3 \\
9\end{array}$ & $\begin{array}{l}+ \\
+\end{array}$ & & $\begin{array}{l}\text { faible-moyen } \\
\text { moyen }\end{array}$ & batch ou continu & plusieurs restrictions \\
\hline $\begin{array}{l}\text { 11. Moussage } \\
\text { ou ébullition }\end{array}$ & & $(+)$ & étalement & faible-moyen & & (aqueuse) \\
\hline 12. Condensation & & + & & & & plusieurs restrictions \\
\hline
\end{tabular}

Récemment, de nouvelles méthodes sont apparues. Il s'agit de systèmes de dispersion par jets (Stang et al., 1994) et de l'émulsification par membrane (Collins et Bowen, 1997). Le système de jet est fondé sur la collision de deux flux préémulsionnés dans une chambre, à la sortie de laquelle l'on obtient l'émulsion. Les mécanismes impliqués sont la turbulence, les forces de cisaillement élongationnelles et la cavitation. Le procédé d'émulsification par membrane consiste à forcer le passage de la phase dispersée vers la phase continue à travers les pores d'une membrane. Les gouttelettes ainsi formées sont détachées de la surface de la membrane par l'écoulement de la phase continue. Les mécanismes de formation de gouttelettes sont tout à fait différents des procédés classiques. La taille et la distribution de tailles des gouttelettes sont déterminées par la taille et la distribution de tailles des pores de la membrane.

Il est important de mentionner également l'existence de méthodes d'émulsification qui nécessitent peu ou pas d'énergie mécanique, comme l'inversion de phase ou l'émulsification spontanée. L'inversion de phase (Schramm, 1992) permet de fabriquer une émulsion fine eau dans huile à partir d'une émulsion huile dans eau grossière (ou inversement) en augmentant progressivement la quantité de phase dispersée, jusqu'à une fraction volumique critique où l'émulsion va soudainement s'inverser. L'émulsification spontanée, connue depuis 1878, est appelée également autoémulsification et ne requiert aucune agitation mécanique (Gopal, 1968). 
Nous avons indiqué dans le tableau 2 les principaux mécanismes impliqués dans chacune des techniques. Les gouttelettes peuvent en effet se déformer et se rompre sous l'effet des forces visqueuses ou d'inertie. Les forces visqueuses génèrent des contraintes tangentielles ou perpendiculaires à la surface de la goutte. Les forces d'inertie, quant à elles, génèrent des différences de pression. En pratique, on distingue les trois cas suivants: écoulement laminaire, écoulement turbulent et cavitation.

Dans un écoulement laminaire, les forces visqueuses sont prédominantes. En écoulement turbulent, ce sont principalement les forces d'inertie qui entrent en jeu, mais les forces visqueuses ne sont pas négligeables. Pendant la cavitation, de petites bulles de vapeur se forment et disparaissent très rapidement, ce qui entraîne l'apparition d'ondes de choc dans la phase continue. Le liquide est alors fortement agité et il apparaît des zones de turbulence.

L'apparition ou la prépondérance de chacun de ces mécanismes ne dépend pas uniquement du type d'appareil utilisé pour préparer l'émulsion. Il faut prendre en compte la viscosité de la phase continue mais également l'échelle de l'appareil (laboratoire ou industriel). Si l'on prend le cas de l'homogénéisateur, le mécanisme principal impliqué dans l'émulsification peut être l'élongation en écoulement laminaire, la turbulence ou la cavitation, suivant la taille, la pression ou la conception de la machine. Par conséquent, il faut garder à l'esprit qu'il est souvent très difficile de prédire à partir d'essais de laboratoire les performances d'un appareil de production.

Il est intéressant à ce niveau de noter qu'un autre mécanisme peut être impliqué dans le processus d'émulsification. Il s'agit de l'instabilité interfaciale due aux gradients de tension interfaciale (Lucassen-Reynders et Kuijpers, 1992). Ce mécanisme dépend essentiellement de la nature des tensioactifs utilisés et peut apparaître dans toutes les techniques de fabrication.

Signalons enfin que différentes variables opératoires ont une importance considérable sur les performances des techniques d'émulsification: la durée de l'agitation (pour les fabrications en batch), la composition du mélange (fraction volumique de la phase dispersée, nature et concentrations des agents tensioactifs, viscosité des deux phases en présence, etc.), et l'ordre d'addition des ingrédients.

\section{FORMATION DES GOUTTELETTES}

\subsection{Formation du film}

Dans la première phase de préparation de l'émulsion, deux phases volumiques sont en présence. Pour que le processus de mise en émulsion commence, il faut qu'il y ait tout d'abord formation d'un film de la «future» phase continue autour de l'autre phase. Ce film doit exister pendant un court instant, mais si aucun tensioactif n'est présent, il sera très instable. Il aura tendance à être drainé très rapidement sous l'action de la gravité. De plus, à l'extrémité de ce film, l'interface se trouvera fortement courbée et les forces interfaciales auront tendance à la redresser.

Par conséquent, ces films ne peuvent exister que si un tensioactif est présent. La stabilité des films a été beaucoup étudiée dans le cas des mousses (interface liquide/air) (Lucassen, 1979). Ce phénomène a été peu étudié dans le cas des émulsions, mais quelques analogies peuvent être faites avec les mousses.

Le principal effet du tensioactif est de permettre l'existence d'un gradient de tension interfaciale $(\mathrm{d} \gamma / \mathrm{d} z)$. Ce gradient exerce une contrainte tangentielle sur le liquide. Lors de la formation de l'émulsion, la génération du gradient de tension interfaciale entraîne une expansion ou une compression locale de l'interface. Les molécules de tensioactif vont alors diffuser à travers l'interface pour tenter de restaurer une tension interfaciale faible. L'écart à l'équilibre de la valeur de la tension interfaciale peut s'exprimer d'après le module de dilatation de surface $E=\mathrm{d} \gamma / \mathrm{d}(\ln A)$. Ce module dépend de la nature du tensioactif, de la vitesse de transport vers l'interface, et du taux d'expansion ou de compression de l'interface. Dans le cas d'un film mince, c'est la quantité de tensioactif présente qui est le facteur déterminant; l'élasticité de Gibbs du film est alors donnée par l'expression:

$$
E_{f}=\frac{2 \mathrm{~d} \gamma / \mathrm{d}(\ln \Gamma)}{1+(1 / 2) h(\mathrm{~d} m / \mathrm{d} \Gamma)}
$$

avec $\Gamma$ concentration en excès du tensioactif $\left(\mathrm{mol} \cdot \mathrm{m}^{-2}\right)$ et $h$ épaisseur du film. Plus le film est mince, plus $E_{f}$ est élevée, et plus le drainage du film est empêché.

L'élasticité de Gibbs joue un autre rôle très important. Le film est la plupart du temps étiré, et en présence d'une quantité suffisante de tensioactif, la partie la plus mince aura l'élasticité la plus élevée, donc une plus grande résistance à l'étirement. Ce phénomène entraîne un mécanisme de stabilisation relativement fort, comme cela a déjà été expliqué par Gibbs (Walstra, 1983). Cependant, dans le cas des mousses, Lucassen (1979) a relevé que $E_{f}$ est égale à zéro quand la concentration en tensioactif $(m)$ est nulle, et passe par un maximum lorsqu'on augmente cette concentration. Par conséquent, quand la concentration est très faible, l'étirement du film entraîne une diminution de l'excès de surface $\Gamma$ et $E_{f}$ peut être très faible dans la partie la plus mince du film; le film peut alors s'avérer très instable. L'une des principales implications est donc que la concentration en tensioactif ne doit pas être trop faible, sinon la formation rapide du film provoque également un épuisement rapide de la quantité de tensioactif dans le film.

Dans le raisonnement développé, deux hypothèses de travail ont été implicitement retenues. On a supposé que le tensioactif était dans la phase continue. S'il se trouvait dans la 
phase dispersée, $E_{f}$ serait toujours très faible et le film instable, ce qui est cohérent avec la règle de Bancroft. La deuxième hypothèse est que l'équilibre de Gibbs entre le tensioactif dans le volume et à l'interface soit atteint. Cette hypothèse n'est pas vérifiée quand on utilise des polymères et, dans ce cas, il est difficile de prévoir l'élasticité de Gibbs.

Cette réflexion sur la formation des films montre clairement que les gradients de tension interfaciale sont absolument nécessaires à la formation des émulsions. La formation des gouttelettes semble facilitée par la formation de films à vitesse modérée. Il semble donc recommandé de commencer une mise en émulsion par une faible agitation.

\subsection{Déformation d'une interface plane}

Nous allons nous intéresser ici à la formation des gouttelettes à partir de l'agitation de deux phases volumiques initialement en contact par une interface plane. Ces deux phases ont des masses volumiques $\rho_{1}$ et $\rho_{2}\left(\rho_{1}>\rho_{2}\right)$, des viscosités $\eta_{1}$ et $\eta_{2}$, et une tension interfaciale $\gamma$ faible. Les principaux mécanismes recensés pour déformer l'interface et provoquer la formation de gouttelettes sont la turbulence, les ondulations capillaires, les instabilités de Rayleigh-Taylor et celles de Kelvin-Helmholtz.

\subsubsection{Turbulence}

Les tourbillons turbulents peuvent être responsables de la destruction de l'interface (Davies, 1972). Des tourbillons de vitesse $u$ peuvent entraîner des pressions locales de l'ordre de $\rho u^{2}$. Des différences de pression de l'ordre de $\left(\rho_{1}-\rho_{2}\right) u^{2}$ de part et d'autre de l'interface peuvent surpasser la pression de Laplace $2 \gamma / R$. Si la taille du tourbillon est de l'ordre de deux fois le rayon de courbure $R$, l'interface peut être divisée. Les calculs réalisés dans les cas de turbulence isotrope montrent que l'interface n'est détruite par la turbulence que si $\gamma$ est très faible (Davies, 1972).

\subsubsection{Ondulations capillaires}

Toute perturbation de l'interface provoque l'apparition de vagues, ou ondulations, qui peuvent dans certains cas amener la formation de gouttelettes. Le problème du calcul des propriétés de ces ondes de surface est bien sûr particulièrement difficile, mais a été résolu dans le cas des petites amplitudes (Lucassen-Reynders et Lucassen, 1969). Dans le cas d'ondes transversales monodimensionnelles, l'équation de Kelvin donne la relation entre la longueur d'onde $(\lambda=2 \pi / k)$ et la fréquence $(v=\omega / 2 \pi)$ :

$$
\omega^{2}\left(\rho_{1}+\rho_{2}\right)=g\left(\rho_{1}-\rho_{2}\right) k+\gamma k^{3}
$$

Le premier terme à droite de l'équation est dû à la gravité et peut être négligé dans notre cas. Le second terme est lié aux forces interfaciales, d'où le terme ondes capillaires. Les ondes sont généralement amorties, mais si une source extérieure maintient une fréquence adéquate, l'amplitude peut augmenter jusqu'à destruction de l'interface. Les gouttelettes résultantes devraient alors avoir un diamètre de l'ordre de $\lambda / 2$.

L'amortissement des ondes capillaires de faible amplitude est connu. On sait que la viscosité des liquides et la présence de tensioactif accélèrent l'amortissement. Cependant, si comme cela arrive dans la pratique, la concentration en tensioactif varie localement, l'amplitude de l'onde peut augmenter là où $\gamma$ est le plus faible. Cela peut être l'un des mécanismes impliqués dans la formation de gouttelettes pendant l'émulsification.

\subsubsection{Instabilité de Rayleigh-Taylor}

Ce type d'instabilité est observé quand l'interface est accélérée perpendiculairement à son plan et dirigée de la phase légère vers la phase lourde. L'accélération gravitaire doit être prise en compte. L'occurence de ce type d'instabilité dans le processus d'émulsification a été plus particulièrement étudié par Gopal (1968) dans le cas du secouage. Dans le cas de liquides parfaits et de $\gamma$ constante, l'interface est instable si $k^{2}<\left(\rho_{1}-\rho_{2}\right) a / \gamma$, et si l'accélération $a$ possède une direction appropriée. Le maximum d'instabilité a lieu pour une longueur d'onde optimale $\lambda_{\text {opt }}$ donnée par:

$$
k_{\mathrm{opt}}^{2}=\left(\rho_{1}-\rho_{2}\right) a / 3 \gamma
$$

Même pour des tensions interfaciales très basses, la longueur d'onde optimale ne permettrait que la formation de gouttes relativement grosses. Une analyse plus poussée montre qu'un profil d'ondes asymétrique se développe, puisque la phase lourde pénètre sous la forme de «pointes » ou de « doigts » dans la phase légère (fig. 1). Ces « pointes » pourraient se casser spontanément en globules, mais cela nécessiterait au moins quelques dixièmes de seconde. Par conséquent, l'importance de ce type d'instabilité dans le processus d'émulsification est douteuse.

\subsubsection{Instabilité de Kelvin-Helmholtz}

Ce type d'instabilité est observé quand les deux phases se déplacent à deux vitesses différentes $u_{1}$ et $u_{2}$ parallèlement à l'interface (Chandrasekhar, 1961). C'est ce qui provoque, par exemple, la formation de vagues par le vent à la surface de l'eau. L'analyse développée est, comme précédemment, valide pour les liquides parfaits et de tension interfaciale constante. On trouve que toute perturbation est favorisée si:

$$
\begin{gathered}
k>a\left(\rho_{1}^{2}-\rho_{2}^{2}\right) / \rho_{1} \rho_{2}\left(u_{1}-u_{2}\right)^{2} \\
\text { et: } \quad\left(u_{1}-u_{2}\right)^{2}>2\left(\rho_{1}+\rho_{2}\right)\left(a \gamma\left(\rho_{1}-\rho_{2}\right)\right)^{1 / 2} / \rho_{1} \rho_{2}
\end{gathered}
$$

Si la condition (12) n'est pas remplie, les forces de surface amortiront les ondes. Le calcul montre que des perturbations 
de longueur d'onde quelconque sont favorisées pour des écarts de vitesses $\left(u_{1}-u_{2}\right)$ tout à fait raisonnables pour un processus d'émulsification. Il est cependant difficile de prévoir si ces perturbations peuvent conduire in fine à la division des gouttelettes. La viscosité des liquides a tendance à améliorer ces effets, alors que les gradients de tension interfaciale les diminuent.

On voit qu'il est particulièrement difficile de prédire quel mécanisme sera principalement responsable de la formation des gouttes. Il est très probable qu'il s'agisse le plus souvent d'une combinaison plus ou moins complexe de tous les mécanismes que nous venons de décrire.

\subsection{Déformation d'un filet cylindrique}

Dans certains cas, des filets cylindriques de la phase dispersée se forment pendant l'émulsification. La déformation d'une interface plane peut conduire à la formation de « pointes », comme nous l'avons vu précédemment, ou bien un jet de liquide peut directement être injecté dans l'autre phase. On peut également imaginer qu'une grosse goutte soit étirée jusqu'à former un filet. La division de ce filet va alors donner naissance à plusieurs gouttelettes.

\subsubsection{Cylindres stationnaires}

Les déformations subies par un cylindre infini de liquide de rayon $\theta$ dans un autre liquide de volume infini ont été étudiées par Rayleigh (Chandrasekhar, 1961). La plupart des déformations sont amorties et seules les déformations axisymétriques de longueur d'onde $\lambda>2 \pi \theta$ conduisent à des instabilités (fig. 2). L'augmentation de l'amplitude $\alpha$ de la déformation augmente alors avec le temps suivant la relation:

$$
\alpha=\alpha_{0} \exp (\beta t)
$$

et le filet se casse dès que $\alpha=\theta$.

Par conséquent, le temps de rupture est donné par:

$$
\tau_{\text {rup }}=(1 / \beta) \ln \left(\theta / \alpha_{0}\right)
$$

Une analyse de cette instabilité pour $\gamma$ constante et en absence d'effets inertiels montre que:

$$
\beta=\left(\gamma / 2 \eta_{\mathrm{c}} \theta\right) F(b, q)
$$

où $F$ est une fonction compliquée de $b=k \theta$ et $q=\eta_{d} / \eta_{c}$. Il existe une longueur d'onde optimale pour laquelle la déformation est dominante et provoque la rupture finale. Le temps de rupture est alors proportionnel à $q$ et $1 / \gamma$, et le rayon des gouttelettes résultantes est:

$$
r=\theta\left(3 \pi / 2 b_{\text {opt }}\right)^{1 / 3}
$$

Cette théorie a été vérifiée par Rumscheidt et Mason (1962) qui ont constaté l'apparition de gouttelettes satellites entre les grosses gouttes pour $q=0,01$ à 10 et des viscosités supérieures à $1 \mathrm{~Pa} \cdot \mathrm{s}$. Dans le cas de liquides dispersés très visqueux ( $q$ élevé), on peut s'attendre à ce que la rupture du filet entraîne l'apparition de gouttes de tailles très variées.
1

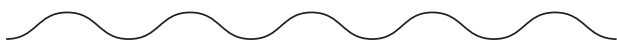

2

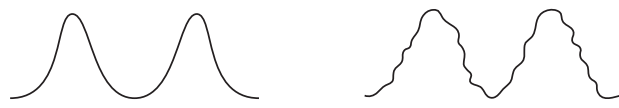

3
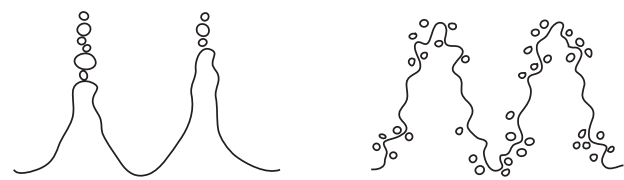

Figure 1

Instabilités de Rayleigh-Taylor; la phase lourde pénètre dans la phase légère; à gauche: instabilités de Rayleigh-Taylor seule; à droite, instabilités de Kelvin-Helmholtz sur les côtés des vagues (d'après Walstra, 1983).

Rayleigh-Taylor instability of interface; the denser phase penetrates into the light phase. At the left side: pure Rayleigh-Taylor instability; at the right side: KelvinHelmholtz instability develops at the flanks of the waves (from Walstra, 1983).

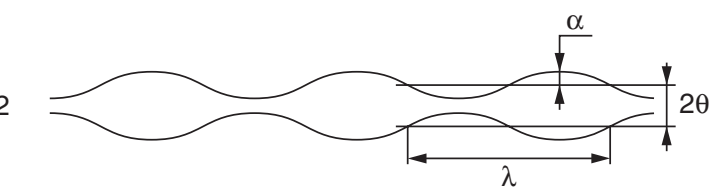

3

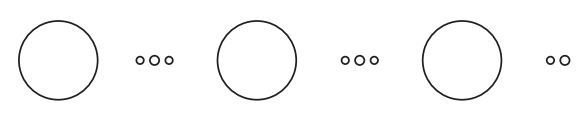

Figure 2

Instabilité d'un cylindre liquide stationnaire.

Instability of a stationary liquid cylinder. 
Le raisonnement précédent, où l'on néglige les effets inertiels, est valable si le nombre de Reynolds est très inférieur à 1 (dans ce cas, $\left.\operatorname{Re}=2 \theta^{2} \beta \rho_{d} / \eta_{d}\right)$. Si Re $>>1$, les effets visqueux peuvent être négligés, et le rayon des gouttes résultantes est égal à 1,9 $\theta$ (cas d'un jet d'eau de quelques millimètres de rayon). Pour les nombres de Reynolds intermédiaires, il n’y a pas de solution évidente.

\subsubsection{Jets issus de restrictions}

Si un cylindre de liquide se déplace à partir d'un orifice dans un autre fluide, avec une vitesse initiale $u$, la stabilité de ce jet n'est pas fondamentalement différente de celle d'un cylindre stationnaire, sauf si $u$ est très faible ou très grande.

Si la vitesse est très faible, les gouttelettes se détachent à partir de l'orifice et leur volume est approximativement égal à $2 \pi \gamma \theta_{0} / g \Delta \rho$, avec $\theta_{0}$, rayon de l'orifice. De nombreux facteurs affectent la taille des gouttes formées, mais des solutions ont été données, y compris pour des liquides non newtoniens (loi de puissance) (Kumar et Saradhy, 1972). Quand la vitesse augmente, la taille des gouttes diminue, et à une certaine vitesse critique (de l'ordre de $\left.\left(\gamma / \rho_{d} \theta_{0}\right)^{1 / 2}\right)$, un jet de liquide se forme avant de se diviser en gouttelettes, et la taille des gouttes est approximativement celle prédite pour les cylindres stationnaires.

Quand la vitesse devient plus importante, le filet de liquide est soumis à des déformations sinusoïdales, sans doute dues aux instabilités de Kelvin-Helmholtz. Des gouttes plus petites sont donc formées dans un temps plus court (Richardson, 1953).

Pour les très grandes vitesses, le jet devient turbulent. Si l'on définit le nombre de Reynolds comme $\operatorname{Re}=2 \theta_{0} u \rho_{d} / \eta_{d}$, le régime turbulent se situe entre 8000 et $10000 . \theta_{0}$ est alors le rayon initial du jet, et ce rayon augmente à mesure que le jet s'éloigne de l'orifice. Le mécanisme de rupture du jet est alors très différent. Les tourbillons turbulents à l'intérieur du jet entraînent la rupture et la résistance visqueuse de la phase continue l'empêche. Par conséquent, $\theta_{0}$ et $\gamma$ n'ont pas d'influence. La distribution de tailles de gouttes peut être très étendue. Le diamètre moyen est donné par la relation suivante (Davies, 1972):

$$
x_{\text {moy }}=3100\left(\eta_{d} / \rho_{d}\right)^{1 / 4}\left(\eta_{c} / \rho_{c}\right)^{3 / 4} u^{-1}
$$

\subsubsection{Filets étirés}

Une goutte dans un liquide qui subit un écoulement axisymétrique élongationnel va s'étirer jusqu'à former un filet. Si le nombre capillaire (rapport des forces visqueuses sur les forces de surface) est suffisamment grand, il y aura rupture par les forces visqueuses:

$$
\mathrm{Ca}=G \eta_{c} r / \gamma
$$

Ce cas a été étudié théoriquement et expérimentalement par Mikami et al. (1975) pour $\gamma$ constante et en absence d'effets inertiels. Si le nombre capillaire n'est pas trop petit, il faut que $\eta_{c}^{2}>\theta \rho_{c} \gamma$; cette condition n'est obtenue que pour des viscosités de la phase continue élevées.

Si l'on suppose que l'on part d'un cylindre non déformé de rayon $\theta_{0}$, le filet va s'étirer selon l'équation:

$$
\theta=\theta_{0} \exp (-G t / 2)
$$

L'extension a un très fort effet d'amortissement sur les instabilités, d'autant plus fort que le nombre capillaire et le rapport de viscosités $q\left(=\eta_{d} / \eta_{c}\right)$ sont élevés. La théorie est relativement compliquée, mais l'on peut retenir que pour $q$ voisin de 1 , le temps de rupture est d'environ $3 / G$ et qu'il augmente avec $q$. Plus le filet pourra être étiré avant sa rupture, plus les gouttes résultantes seront petites.

\subsubsection{Effets des tensioactifs}

Le tensioactif a d'autres effets que simplement abaisser la tension interfaciale $\gamma$. Il permet le développement de gradients de tension interfaciale, qui empêchent le mouvement tangentiel de l'interface, donc l'écoulement des liquides le long de cette interface. Souvent, le tensioactif a tendance à rendre le filet plus stable, mais il ralentit également la vitesse d'amincissement des filets étirés. L'effet global se révèle cependant plutôt faible.

Pendant l'émulsification, les conditions de cisaillement, notamment, varient considérablement $(G)$, et il est particulièrement difficile de faire des prédictions, mais il est raisonnable de penser que la présence d'un tensioactif favorise l'apparition de gouttelettes plus petites. Il est intéressant de noter que des gouttes « satellites » ne sont pas toujours observées en présence de tensioactif (Caroll et Lucassen, 1976).

\section{RUPTURE DES GOUTTELETTES}

Nous allons maintenant nous intéresser à la rupture des gouttelettes sous différents régimes d'écoulement, en laminaire comme en turbulent. Notons qu'une discussion générale sur l'effet des différents modèles d'écoulement sur la déformation et le cassage des gouttelettes a été réalisée par Hinze (1955) et, plus récemment, Stone (1994) a publié un article sur la déformation et la rupture des gouttes dans les fluides visqueux.

En général, le mode de rupture des gouttes peut être appréhendé à partir de la compétition entre deux types de forces (Hinze, 1955): une force externe induite par l'écoulement, qui tend à déformer la goutte, et une force interne de restitution, qui tend à conserver la forme initiale de la goutte. La rupture a lieu lorsque la force de déformation devient trop importante. 
En première approximation, la force interne peut être représentée par le rapport $\gamma / d$ (force de Laplace). La force de déformation $F_{d}$ peut être inertielle ou visqueuse et est exercée par la phase continue sur la goutte. Le rapport entre les deux forces, $F_{d} d / \gamma$, est très souvent utilisé pour décrire les processus de rupture. En général, il est appelé nombre capillaire quand on est en présence de forces visqueuses, comme nous l'avons vu précédemment, ou nombre de Weber lorsque les forces de déformation sont inertielles. De nombreux auteurs emploient cependant indifféremment l'un ou l'autre terme. Si ce rapport excède une certaine valeur, il y a rupture de la goutte.

\section{1 Écoulement laminaire}

Nous allons considérer ici un écoulement laminaire sans effets d'inertie. Une gouttelette va se casser en flux laminaire si son nombre capillaire est supérieur à une valeur critique $\mathrm{Ca}_{\mathrm{cr}}$. Cela veut dire que pour certaines conditions fixées, il existe un rayon de gouttelette critique $r_{\text {cr }}$, pour lequel la goutte va se casser si $r>r_{\text {cr. }}$. Ce nombre capillaire critique dépend essentiellement du type d'écoulement et du rapport de viscosités $q$. Une revue sur la déformation et le cassage des gouttes a été donnée par Acrivos (1974).

\subsubsection{Cisaillement simple}

Le tableau 3 regroupe les hypothèses utilisées dans les principales théories sur la déformation des gouttelettes en écoulement laminaire.

L'analyse classique de Taylor (1934) donne des résultats remarquables, malgré l'hypothèse de négligence de l'effet de déformation sur la contrainte (hypothèse 8 ). La déformation $D$ à l'équilibre est donnée par:

$$
D=(L-B) /(L+B)=\mathrm{Ca}(19 q+16) /(16 q+16)
$$

cela pour des valeurs de $q$ pas trop élevées. $L$ est la dimension la plus grande de la goutte déformée et $B$ la dimension la

TABLEAU 3

Conditions des théories sur la déformation de gouttelettes en écoulement laminaire (d'après Walstra, 1983)

Conditions used in theories about droplet deformation in laminar flow (from Walstra, 1983)

\begin{tabular}{|c|c|c|c|}
\hline Théories & $\begin{array}{l}\text { Taylor } \\
\text { (1934) }\end{array}$ & $\begin{array}{c}\text { Cox } \\
(1969)\end{array}$ & $\begin{array}{l}\text { Barthès-Biesel } \\
\text { et Acrivos* (1973) }\end{array}$ \\
\hline $\begin{array}{l}\text { Types d'écoulement } \\
\text { Cisaillement simple } \\
\text { Hyperbolique plan } \\
\text { Extensionnel axisymétrique }\end{array}$ & $\begin{array}{l}+ \\
+\end{array}$ & + & $\begin{array}{l}+ \\
+ \\
+\end{array}$ \\
\hline $\begin{array}{l}\text { Hypothèses } \\
\text { 1. Écoulement illimité, stationnaire } \\
\text { 2. Liquides incompressibles } \\
\text { 3. Liquides newtoniens } \\
\text { 4. Effets d'inertie négligeables** } \\
\text { 5. Agitation thermique négligeable*** } \\
\text { 6. État stationnaire } \\
\text { 7. } \gamma \text { constante } \\
\text { 8. Contraintes pour une goutte non déformée } \\
\text { 9. Petite déformation } \\
\text { 10. Contrainte tangentielle continue } * * * * \\
\text { 11. Vitesse tangentielle continue**** } \\
\text { 12. Contrainte normale = pression } \\
\text { de Laplace } * * * * * \\
\text { 13. Vitesse normale continue } \\
\text { (3***** }\end{array}$ & $\begin{array}{l}+ \\
+ \\
+ \\
+ \\
+ \\
+ \\
+ \\
+ \\
+ \\
+ \\
+\end{array}$ & $\begin{array}{l}+ \\
+ \\
+ \\
+ \\
+\end{array}$ & $\begin{array}{l}+ \\
+ \\
+ \\
+\end{array}$ \\
\hline
\end{tabular}

\footnotetext{
* Cette théorie inclut l'éclatement des gouttes

** $\quad$ Donc des petites gouttes; pas d'effet de densité

*** Donc des gouttes pas trop petites

**** D'après les hypothèses 4 et 7

****** D'après l'hypothèse 4

****** D'après l'hypothèse 2 et l'équation de continuité
} 
plus petite (fig. 3). On trouve en général que la goutte se casse pour $D>0,5$, ce qui correspond à une extension de la goutte d'environ deux fois son diamètre initial.

Cette théorie a été complétée par Cox (1969). L'évolution de la déformation en fonction du temps est donnée par l'équation:

$$
D(t) \approx D\left(1-\exp \left(-\gamma t / r \eta_{d}\right)\right)
$$

et la déformation finale est:

$$
D=\frac{5(19 q+16)}{4(1+q)\left((19 q)^{2}+(20 / \mathrm{Ca})^{2}\right)^{1 / 2}}
$$

Par conséquent, pour $q$ tendant vers $0, D$ tend vers l'infini comme dans la théorie de Taylor, et pour $q$ tendant vers l'infini, $D$ tend vers 0 . Les gouttes très visqueuses se déforment donc très difficilement.
La théorie de Cox a été encore complétée par BarthèsBiesel et Acrivos (1973) de façon à prendre en compte les déformations importantes et la rupture des gouttes, mais les équations deviennent très compliquées. On trouve en fait que le nombre capillaire critique $\mathrm{Ca}_{\text {cr }}$ est une fonction de $q$ uniquement, et les résultats expérimentaux sont relativement bien en accord avec la théorie. Cependant, suivant les auteurs, on trouve quelques divergences quant aux résultats expérimentaux, divergences inévitables puisque le mode de cassage des gouttelettes dépend également de $\mathrm{d} G / \mathrm{d} t$, en particulier pour les taux de cisaillement élevés (fig. 4a). Par conséquent, il est tout à fait possible de prédire un rayon critique de gouttes de façon relativement précise, mais il est impossible de prévoir la taille des gouttelettes résultantes.

Les principales conclusions concernant la rupture des gouttelettes dans un écoulement de cisaillement simple sont résumées par la figure 5 (nombre capillaire en fonction du rapport des viscosités, d'après Janssen et al., 1994).
Type

d'écoulement

a

Rotation

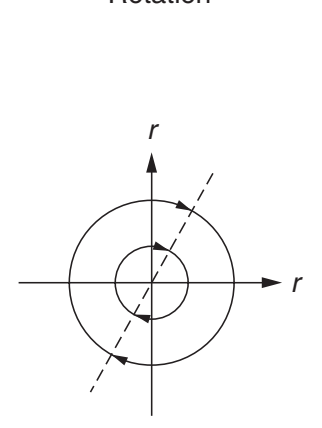

Modèle

d'écoulement

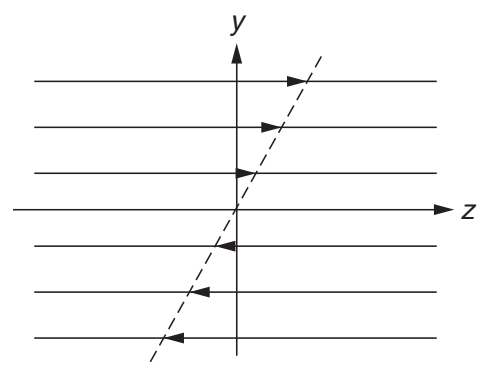

Cisaillement simple

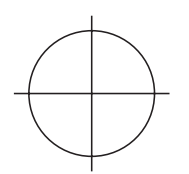

Forme

de la goutte

\section{Gradient}

de vitesse

Vitesse

de rotation $\mathrm{d} u_{\tan } / \mathrm{d} r$

G

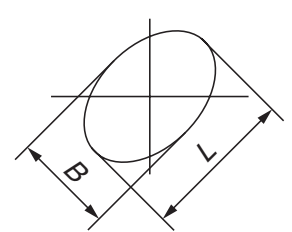

$\mathrm{d} u_{z} / \mathrm{d} y$

G/2 c

Hyperbolique
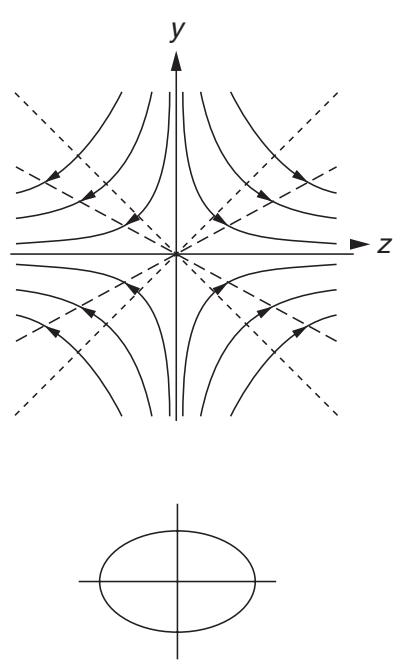

$\mathrm{d} u_{z} / \mathrm{d} z$

Figure 3

Différents types d'écoulements bidimensionnels et leurs effets sur la déformation de gouttes.

Different types of two-dimensional flows and their effect on droplet deformation. 


\begin{tabular}{|c|c|c|}
\hline $\begin{array}{c}\text { Modèle } \\
\text { d'écoulement }\end{array}$ & Conditions & Rupture \\
\hline $\begin{array}{l}\text { a Cisaillement } \\
\text { simple }\end{array}$ & $\mathrm{d} G / \mathrm{d} t=>0$ & $P \rightarrow 0.0 .0$ \\
\hline $\begin{array}{l}\text { b Cisaillement } \\
\text { simple }\end{array}$ & $\begin{array}{l}\mathrm{d} G / \mathrm{d} t \text { élevé } \\
q \text { faible }\end{array}$ & $0 \rightarrow 0$ \\
\hline $\begin{array}{l}\text { c Cisaillement } \\
\text { simple }\end{array}$ & $\begin{array}{l}\mathrm{d} G / \mathrm{d} t \text { élevé } \\
q \approx 1\end{array}$ & $O \rightarrow \rightleftharpoons$ \\
\hline $\begin{array}{l}\text { d Hyperbolique } \\
\text { plan }\end{array}$ & $q$ faible & $\bigcirc \rightarrow$ \\
\hline $\begin{array}{l}\text { e Hyperbolique } \\
\text { plan }\end{array}$ & $q$ élevé & \\
\hline
\end{tabular}

Figure 4

Rupture de gouttelettes sous différentes conditions d'écoulement (d'après Rumscheidt et Mason, 1962).

Droplet break-up under various flow conditions (from Rumscheidt and Mason, 1962).

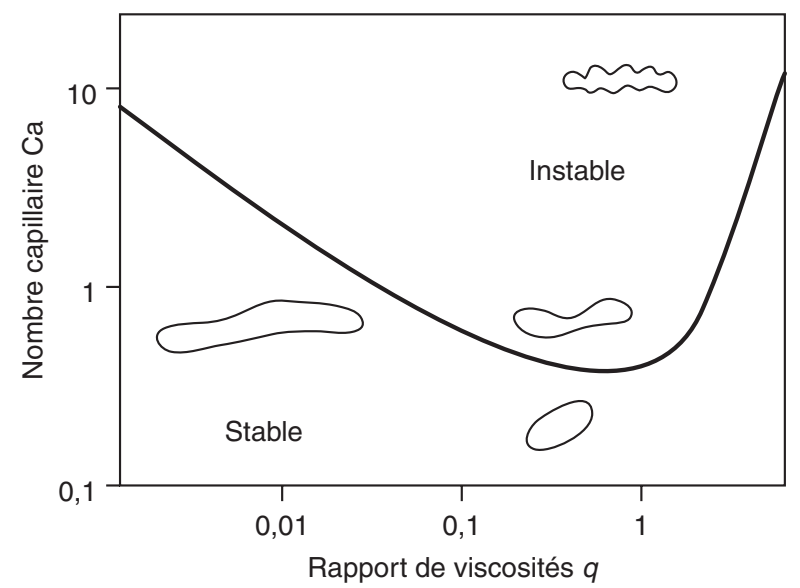

Figure 5

Cassage de gouttelettes en écoulement de cisaillement simple (d'après Janssen et al., 1994).

Droplet break-up under simple shear flow (from Janssen et al., 1994).

\subsection{2 Écoulement élongationnel}

Les effets de l'écoulement hyperbolique plan ont été étudiés à la fois théoriquement (tableau 3) et expérimentalement (Grace, 1982). Les expériences sont généralement réalisées dans un appareil contenant quatre cylindres en rotation provoquant un écoulement, comme montré sur la figure $3 \mathrm{c}$. On constate en général que le nombre de Weber critique est plus petit que dans le cas d'un cisaillement simple. On peut également observer un éclatement de la goutte pour les rapports de viscosités $q$ élevés. Cet éclatement ne correspond pas forcément à une division de la goutte en deux gouttelettes (fig. $4 d$ et 4 e).

Il faut cependant bien avoir à l'esprit que, contrairement à la rupture sous cisaillement simple, la rupture sous cisaillement élongationnel est efficace pour des rapports de viscosité de la phase dispersée sur la viscosité de l'émulsion allant de 0,01 à 100 (Grace, 1982).

\subsection{3 Écoulement de Poiseuille}

Dans ce type d'écoulement, on observe un profil de vitesse parabolique. $G$ est proportionnel à la distance par rapport au centre du tube. Si le rayon de la goutte est très petit devant le diamètre du tube, on peut appliquer la théorie développée en cisaillement simple, en prenant la valeur de $G$ au centre de gravité de la goutte. En revanche, si la taille de la goutte se rapproche de la valeur du diamètre du tube, la goutte se déplace au centre du courant et se déforme (fig. 6).
La déformation d'une goutte dans un écoulement de Poiseuille entre deux plaques parallèles a été étudiée par Kiefer (1977). En négligeant les forces visqueuses, il donne une expression de la déformation finale:

$$
\left(z_{1}+z_{2}\right) / 2 r \approx\left(y_{0} / r\right)^{2} \rho_{c} u^{2}(r / h)^{4}(r / \gamma)
$$

En première approximation, la rupture de la goutte a lieu si:

$$
\left(\rho_{c} u^{2} r^{5} / h^{4} \gamma\right)>2
$$

où $u$ est la vitesse moyenne du liquide, et, comme $2 r<h$, si :

$$
\left(\rho_{c} u^{2} h / \gamma\right)>64
$$

Cette théorie s'applique surtout pour des valeurs de $q$ élevées, de faibles viscosités de la phase continue et des $h$ très petites.

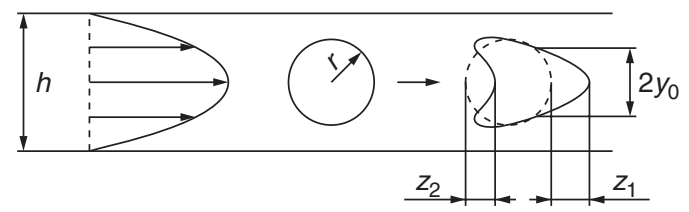

Figure 6

Déformation d'une gouttelette en écoulement de Poiseuille. Droplet deformation under Poiseuille flow. 
Rappelons que toutes ces études ont été réalisées en considérant des liquides newtoniens. Il est évident que dans la réalité, on peut se trouver en présence de comportements un peu plus compliqués. Le fluide peut avoir un comportement rhéofluidifiant (la viscosité apparente diminue quand le taux de cisaillement augmente), il peut présenter une contrainte seuil (pas d'écoulement en deçà d'un certain taux de cisaillement), ou bien il peut être viscoélastique (fluides à mémoire) et montrer également un comportement rhéofluidifiant.

Les liquides uniquement rhéofluidifiants (appelés également purement visqueux) peuvent provoquer dans les cas extrêmes des écoulements avec «effet bouchon». Cela arrive surtout avec les dispersions.

Dans le cas des liquides viscoélastiques, le problème devient beaucoup plus compliqué. Il faut prendre en compte le temps caractéristique de relaxation $\tau_{\text {mem }}$ (pour lequel on observe une disparition de la contrainte élastique). Un article sur le déplacement, la déformation et l'éclatement de gouttes dans les liquides viscoélastiques a été publié par Zana et Leal (1974). Dans une première approche, on peut dire que, si la phase dispersée est viscoélastique, la rupture des gouttelettes est plus difficile, surtout si le temps de relaxation est grand. Dans le cas d'une phase continue viscoélastique, il semble très difficile d'obtenir des gradients de vitesse élongationnels élevés (Walstra, 1993). Cependant, Mason et Bibette (1996, 1997) ont récemment montré qu'il est très facile de fabriquer des émulsions monodisperses avec très peu d'énergie, par simple cisaillement, à partir de fluides complexes viscoélastiques. Le principe consiste à fabriquer une première émulsion grossière et à lui appliquer un cisaillement régulier (entre deux plaques circulaires ou dans une géométrie du type Couette) ou un cisaillement oscillant, avec un entrefer très petit (inférieur à $200 \mu \mathrm{m}$ ). Les auteurs attribuent ce résultat à une modification de l'instabilité capillaire ordinaire due à l'élasticité partielle du fluide. Cette élasticité conduirait à une longueur d'onde hautement sélective, entraînant une monodispersité accrue de l'émulsion finale.

\subsubsection{Application à l'émulsification}

La théorie de rupture des gouttelettes en conditions d'écoulement laminaire peut, avec quelques réserves, être appliquée aux moulins à colloïdes (cisaillement simple) pour des viscosités de phase continue pas trop faibles, et à des écoulements à travers des restrictions (écoulement extensionnel axisymétrique) pour $q<4$. Il est évident qu'il y a peu d'appareillages capables de préparer une émulsion stable en conditions laminaires. La théorie a cependant été testée avec un moulin à colloïdes, utilisé dans des conditions de cisaillement simple. Les auteurs ont fait varier la composition des deux phases ainsi que la concentration en tensioactif (Ambruster, 1990; Schubert et Ambruster, 1989). Le diamètre moyen des gouttelettes (diamètre de Sauter, $d_{32}$ ) a été déterminé et utilisé pour calculer le nombre capillaire critique $\mathrm{Ca}_{\text {cr }}$. Les auteurs ont constaté un accord satisfaisant avec la théorie (fig. 7).

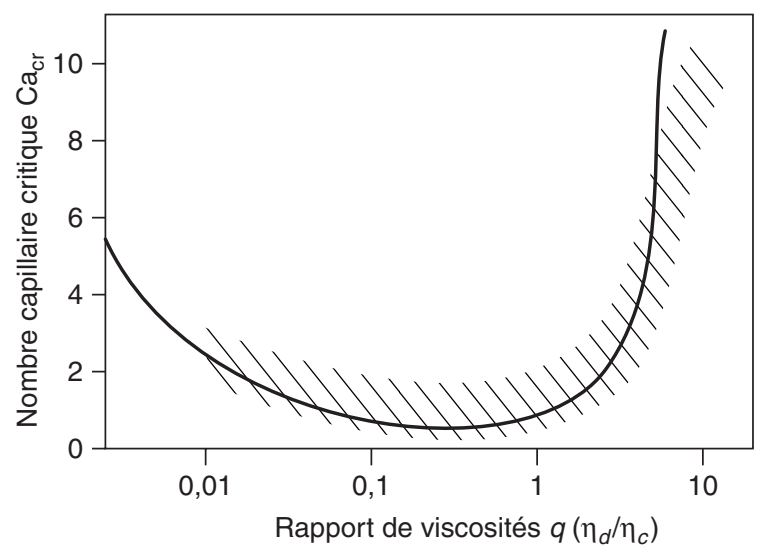

Figure 7

Comparaison entre la courbe théorique du nombre capillaire critique en cisaillement simple (courbe pleine d'après Grace, 1982) et les résultats expérimentaux d'Ambruster (1990) obtenus avec un moulin à colloïdes (hachures).

Comparison between the theoretical curve of capillary number versus viscosity ratio under simple shear flow (from Grace, 1982) and the experimental results from Ambruster (1990) obtained with a colloid mill (hatching).

\section{2 Écoulement turbulent}

\subsubsection{La turbulence}

Il est clair, d'après ce que nous avons vu, que l'écoulement laminaire ne permet pas de casser des gouttelettes dispersées dans l'eau ou dans un autre liquide de faible viscosité. Les conditions d'écoulement doivent alors être hautement turbulentes, entraînant la plupart du temps une prédominance des forces inertielles. En écoulement turbulent (Davies, 1972), la vitesse locale $u$ varie de façon chaotique. Les déviations sont caractérisées par $\Delta u=\sqrt{\left(u-u_{\text {moy }}\right)^{2}}$, avec $u_{\text {moy }}$, vitesse moyenne. L'écoulement est isotrope si $\Delta u$ est la même dans toutes les directions.

Seul l'écoulement turbulent isotrope peut être caractérisé de façon relativement simple, mais ce type d'écoulement est très difficile à obtenir pratiquement. Les grands tourbillons en écoulement turbulent, dont la taille $x_{e}$ est de l'ordre de grandeur du diamètre du tube par exemple, ont une vitesse $\Delta u$ plutôt faible; ils transfèrent donc leur énergie cinétique aux tourbillons plus petits et ainsi de suite. Il existe donc un spectre de tailles de tourbillon. Les plus petits tourbillons $(\Delta u$ élevées et de taille $x_{0}$ ) voient leur énergie cinétique dissipée en chaleur. La dimension des plus petits tourbillons est appelée échelle de Kolmogorov et les gouttes de diamètre inférieur à ces tourbillons sont généralement très peu déformées :

$$
x_{0}=\eta_{c}^{3 / 4} \rho_{c}^{-1 / 2} \varepsilon^{-1 / 4}
$$


Les tourbillons un peu plus importants (dits porteurs d'énergie) sont en grande partie responsables de la rupture des gouttelettes, et sont caractérisés par:

$$
\Delta u(x)=C x^{1 / 3} \varepsilon^{1 / 3} \rho_{c}^{-1 / 3}
$$

où $x$ est la taille du tourbillon et $C$ une constante de l'ordre de l'unité. La densité de puissance $\varepsilon$ est la quantité moyenne d'énergie dissipée par unité de temps et de volume. Le temps caractéristique d'un tourbillon est:

$$
\tau(x)=C x^{2 / 3} \varepsilon^{-1 / 3} \rho^{1 / 3}
$$

\subsubsection{Taille des gouttelettes}

La théorie qui définit les conditions gouvernant la taille des gouttelettes en écoulement turbulent est largement due à Kolmogorov (Levich, 1962) et Hinze (1955).

Le mécanisme de rupture des gouttelettes en écoulement turbulent va dépendre du nombre de Reynolds des gouttes $\left(\operatorname{Re}_{g}\right)$. Si ce nombre est supérieur à 1 (pour de faibles $\eta_{c}$ ), ce sont les forces d'inertie qui prédominent. En revanche, si $\operatorname{Re}_{g}$ est inférieur à 1 , les forces de cisaillement entrent en jeu, et le type d'écoulement local rencontré est essentiellement élongationnel.

Plaçons-nous d'abord dans le cas où les forces d'inertie agissent $\left(\operatorname{Re}_{g}>1\right)$. Les tourbillons créent des différences de pression (de l'ordre de $\left.\rho_{c}(\Delta u(x))^{2}\right)$ qui, si elles sont supérieures à la pression de Laplace, peuvent entraîner la rupture d'une goutte de diamètre $x$ se trouvant dans leur voisinage. Le nombre de Weber de la goutte est donné par l'expression suivante:

$$
\mathrm{We}=\rho_{c} \Delta u^{2} d / \gamma
$$

Par conséquent, en combinant les équation (27) et (29), on obtient le diamètre maximal des gouttelettes dans le champ turbulent:

$$
d_{\max }=x_{\max }=C \varepsilon^{-2 / 5} \gamma^{3 / 5} \rho_{c}^{-1 / 5}
$$

Il est évident que le champ de turbulence est rarement homogène et que la distribution de tailles des gouttelettes peut être très étendue. Cependant, cette équation s'avère très utile et permet de prédire correctement la taille des plus grandes gouttes, à condition toutefois que la recoalescence soit limitée. Cette équation peut également décrire (avec une constante différente) le diamètre moyen des gouttelettes. Bien sûr, un grand nombre de conditions doivent être remplies (Walstra, 1983): nombre de Reynolds élevé, $x_{\max }>x_{0}$, nombre de Reynolds de la goutte $\operatorname{Re}_{g}>5$. Si l'écoulement au voisinage de la goutte devient laminaire, l'équation (27) ne s'applique plus localement, et la rupture par les forces d'inertie est impossible. En combinant cette condition avec $\mathrm{We}_{\mathrm{cr}}=1$, on obtient la taille minimale de goutte à laquelle l'équation (30) peut s'appliquer:

$$
x_{\min } \approx 3 \eta_{c}^{2} / \gamma \rho_{c}
$$

Une autre condition est que le temps de déformation $\tau_{\text {déf }}$ soit inférieur au temps caractéristique du tourbillon $\tau\left(x_{\max }\right)$.

L'équation (30) prédit que le changement de viscosité de la phase continue n'affectera pas la taille des gouttelettes. On observe cependant expérimentalement une légère dépendance, la taille moyenne des gouttelettes diminuant lorsque la viscosité augmente. Cela est lié au fait que les fluctuations de pression ne sont pas toujours la seule cause de rupture des gouttes. Pour une goutte située entre deux tourbillons de taille très supérieure à celle de la goutte, les forces de cisaillement doivent agir et provoquer cette rupture. On se trouve alors dans le cas où $\mathrm{Re}_{g}$ est inférieur à 1 . On suppose que ce type d'écoulement est similaire à un écoulement hyperbolique plan. On obtient donc dans ce cas:

$$
d_{\max }=C \gamma \varepsilon^{-1 / 2} \eta_{c}^{-1 / 2}
$$

Si l'on se trouve dans une situation intermédiaire, la viscosité de la phase continue pourrait donc avoir un effet. Cependant, des travaux récents, menés par Stang et al. (1997) sur des moulins à colloïdes, ont montré qu'à densité d'énergie constante, la viscosité de la phase continue n'a pas d'influence sur la rupture des gouttelettes, à condition qu'un émulsifiant « rapide » soit utilisé. En présence d'émulsifiants « lents » (qui s'adsorbent lentement à l'interface), selon la nature du stabilisant, la taille des gouttes est susceptible d'augmenter avec la viscosité de la phase continue, à énergie volumique constante, cela en raison de phénomènes de coalescence.

Si l'on ajoute un polymère à la phase continue, cela provoque une augmentation de la viscosité, mais aussi une diminution de la turbulence; en particulier, on n'observe plus les plus petits tourbillons. On obtient donc une taille moyenne de gouttelettes plus élevée et une distribution plus serrée.

Enfin, l'équation (30) ne prédit pas d'effet de la viscosité de la phase dispersée sur la taille des gouttes, ce qui n'est pas vérifié expérimentalement (Walstra, 1974). Dans des expériences avec des émulsions d'huile de paraffine dans l'eau, fabriquées avec divers surfactifs et différents appareils, il a été observé que le diamètre moyen $x_{43}$ était proportionnel à $\eta_{d}^{0,37}$ à $\varepsilon$ constante. Cet effet de viscosité a été discuté par Davies (1985) qui a modifié l'équation (30) en ajoutant un terme lié aux contraintes visqueuses $\left(\left(\gamma+\eta_{d} \Delta u / 4\right)\right.$ à la place de $\gamma)$. Cette équation ne permet cependant pas de décrire correctement les observations expérimentales. Il apparaît alors utile de comparer le temps de vie du tourbillon (éq. (28)) et le temps de déformation (Walstra, 1993):

$$
\tau_{\text {déf }} \approx \eta_{d} /\left(C \varepsilon^{2 / 3} d^{2 / 3} \rho^{1 / 3}-4 \gamma / d\right)
$$

Cette équation est différente de celle donnée précédemment (éq. (3)) mais est plus réaliste. Par conséquent, pour $\eta_{d}$ faible, le temps de déformation est plus petit que le temps de vie des tourbillons de la taille de la goutte, ce qui implique que les fluctuations de pression doivent être suffisantes pour 
que les gouttelettes soient cassées par ces tourbillons. Ce n'est plus le cas pour des gouttelettes de viscosité plus élevée. Pour des $\eta_{d}$ plus élevées, les gouttelettes résultantes sont donc en moyenne plus grosses et leur distribution plus étendue, ce qui est en effet observé expérimentalement (Walstra, 1974; Schubert et Stang, 1997). Plusieurs auteurs ont tenté de prendre en compte l'effet de la viscosité de la phase dispersée dans l'expression du diamètre maximal (Davies, 1985; Sleicher, 1962; Hinze, 1955). D'autres auteurs utilisent le nombre d'Ohnsorge, $\mathrm{Oh}=\eta_{d} /\left(\gamma \rho_{d} d\right)^{1 / 2}$, et proposent les relations suivantes (Karbstein et Schubert, 1995):

- si Oh est faible $\left(\eta_{d}<10 \mathrm{mPa} \cdot \mathrm{s}\right): d_{\max } \approx \varepsilon^{-2 / 5}$

- si Oh est élevé $\left(\eta_{d}>>10 \mathrm{mPa} \cdot \mathrm{s}\right): d_{\max } \approx \varepsilon^{-1 / 4} \eta_{d}^{3 / 4}$

\subsubsection{Application aux différents appareils}

De nombreuses études ont été effectuées pour valider l'équation (30), le plus souvent dans des réacteurs agités. Certains auteurs n'ont pas ajouté de tensioactif et ont utilisé diverses techniques pour empêcher la coalescence avant analyse. Walstra (1983) a analysé les différents résultats obtenus. Nous avons regroupé dans le tableau 4 les relations issues de l'équation (30) obtenues pour différents types d'appareils utilisés en écoulement turbulent.

\section{TABLEAU 4}

Prédiction du diamètre maximal des gouttes en fonction de différents appareils (d'après Walstra, 1983)

Prediction of the maximum droplet diameter for different apparatuses (from Walstra, 1983)

\begin{tabular}{lc}
\hline \multicolumn{1}{c}{ Appareils } & $d_{\max }$ \\
\hline Agitateurs & $C v^{-6 / 5} X^{-4 / 5} \rho_{c}^{-3 / 5} \gamma^{3 / 5}$ \\
1. À turbine & $C \approx 0,1$ \\
2. Cylindres coaxiaux & $C \approx 0,73$ \\
3. Rotor-stator (ultra-turrax) & $C \approx 1,3$ \\
\hline Écoulement en conduite & $C \theta^{2 / 5} \gamma^{3 / 5} \rho^{-3 / 5} u^{6 / 5}$ \\
\hline Homogénéisateurs & $C p_{h}^{-3 / 5} \gamma^{3 / 5} z_{d}^{2 / 5}$ \\
\hline$C=$ constante & $v=$ vitesse de révolution \\
$X=$ diamètre de l'agitateur & $\theta=$ rayon de la conduite \\
$p_{h}=$ pression (quantité d'énergie dissipée par unité de volume) \\
$z_{d}=$ distance effective sur laquelle l'énergie est dissipée
\end{tabular}

Pour aboutir à ces relations, il suffit de remplacer $\varepsilon$ dans l'équation (30). Pour un écoulement en conduite, $\varepsilon$ est de l'ordre de $\rho u^{3} / \theta$. Dans le cas des homogénéisateurs, elle est proportionnelle à $p_{h}\left(u / z_{d}\right)$ avec $u$ de l'ordre de $\sqrt{P_{h} / \rho}$. Enfin, dans le cas des agitateurs, la densité de puissance $\varepsilon$ est proportionnelle à $\rho v^{3} X^{2}$. L'équation dérivée (éq. (34)) est dite relation de Hinze-Clay. Certains auteurs préfèrent la présenter sous une forme un peu différente, qui fait appel à un nombre adimensionnel, le nombre de Weber de l'agitateur (à ne pas confondre avec le nombre de Weber de la goutte) (Desnoyer et al., 1998):

$$
d_{\max } / X \text { ou } d_{32} / X \approx\left(\mathrm{We}_{\text {agitateur }}\right)^{-0,6}
$$

avec $\mathrm{We}=\rho v^{2} X^{3} / \gamma$.

\subsection{Cassage par cavitation}

La cavitation est un phénomène lié à la formation soudaine de cavités, qui disparaissent aussitôt, comme par exemple des bulles de vapeur ou de gaz dans un liquide. Cela peut arriver quand la pression locale descend en dessous de la pression de vapeur. La cavitation est donc un processus complexe qui se déroule en trois étapes: nucléation, croissance et effondrement des bulles de gaz ou de vapeur. Les variations de pression nécessaires à une nucléation homogène sont particulièrement importantes. La plupart du temps, on est plutôt en présence d'une nucléation hétérogène de nature très aléatoire, puisque dépendante des impuretés du liquide qui vont servir de catalyseur.

Des pressions faibles peuvent être créées en infligeant au liquide des vitesses élevées ou en déplaçant un objet à très grande vitesse au sein du liquide. On peut générer une cavitation contrôlée par des ondes sonores, en particulier avec des ultrasons. Une bonne revue à ce sujet a été donnée par Basedow et Ebert (1977).

Les cavités obtenues par ultrasons peuvent être transitoires ou oscillantes. Ce sont les cavités transitoires qui sont les plus intéressantes pour l'émulsification car elles s'effondrent aussitôt après leur formation. Cet effondrement a lieu quand la fréquence utilisée est inférieure à la fréquence naturelle des cavités (> $20 \mathrm{kHz}$ pour l'eau). Pour l'émulsification dans l'eau, on utilise donc souvent des fréquences de $20 \mathrm{kHz}$. La disparition des cavités a lieu très rapidement, dans un ordre de temps inférieur à l'inverse de la fréquence utilisée. L'effondrement rapide des cavités produit localement une pression élevée $\left(10^{8}\right.$ à $\left.10^{11} \mathrm{~Pa}\right)$, qui augmente quand la fréquence diminue. Une toute petite onde de choc de très grande intensité est alors générée, ce qui entraîne, en présence d'une particule quelconque, l'existence d'une densité d'énergie très élevée, dans un espace très réduit et pendant un temps très court. Ce phénomène de cavitation peut engendrer différents effets, dont la rupture de gouttelettes d'émulsion.

Il semble que le mécanisme exact de rupture des gouttelettes dans le cas de l'émulsification ultrasonique n'est pas identifié (Walstra, 1983). Les résultats d'expériences menées au laboratoire ne sont pas toujours reproductibles.

En ce qui concerne les homogénéisateurs haute pression, il a été reporté que dans certains cas, la cavitation est responsable de la rupture des gouttelettes (Kurzhals, 1977). Cependant, dans la plupart des homogénéisateurs, l'énergie due aux ultrasons ne représente que quelques pour-cent de l'énergie totale dissipée, essentiellement apportée par la turbulence. 


\section{RÔLE DES TENSIOACTIFS}

Les agents tensioactifs jouent des rôles très variés dans le processus d'émulsification. Certains rôles sont bien identifiés, d'autres restent sujets à controverse.

\subsection{Abaissement de la tension interfaciale}

C'est évidemment la première fonction qui vient à l'esprit. La tension interfaciale entre la plupart des huiles et l'eau est de l'ordre de 30 à $50 \mathrm{mN} / \mathrm{m}$. L'addition de polymères tensioactifs peut faire descendre cette valeur à $10 \mathrm{mN} / \mathrm{m}$. De bons émulsifiants permettent d'atteindre $1 \mathrm{mN} / \mathrm{m}$, et certains mélanges tensioactif-cotensioactif utilisés à forte concentration donnent des tensions interfaciales de quelques $\mu \mathrm{N} / \mathrm{m}$.

Cependant, toutes ces valeurs sont données à l'équilibre. Durant l'émulsification, la tension interfaciale à la surface des gouttelettes peut être beaucoup plus élevée que la valeur d'équilibre (Janssen et al., 1994). En effet, l'adsorption du tensioactif sur une grande interface entraîne la diminution de sa concentration dans le liquide environnant, et de plus, le temps d'adsorption peut être relativement long comparé au temps de rupture des gouttelettes et au temps de collision. Par conséquent, l'abaissement de la tension interfaciale n'est pas le rôle principal du tensioactif pendant le processus d'émulsification.

\subsection{Action sur le mode de déformation}

Cette action est liée à la formation de gradients de tension interfaciale le long de l'interface. En absence de tensioactif, le mouvement du fluide est continu de part et d'autre de l'interface. En présence de tensioactif, l'écoulement du fluide le long de l'interface crée un gradient de tension interfaciale qui, s'il est suffisamment élevé, peut immobiliser la surface. L'apparition d'un gradient de tension interfaciale sur une surface initialement immobile peut également provoquer l'écoulement: c'est l'effet Marangoni (fig. 8).

Par conséquent, la présence de tensioactif entraîne une variation locale de la valeur de la tension interfaciale, ainsi qu'un mouvement latéral de l'interface. De plus, le tensioactif empêche la circulation du liquide dans la goutte. Enfin, il peut apporter une résistance visqueuse supplémentaire à l'agrandissement de l'interface (Lucassen-Reynders et Kuijpers, 1992). Il est évident que ces effets vont affecter le mode de rupture ainsi que la taille des gouttelettes.

Si la tension interfaciale est très faible, l'interface peut devenir instable à cause des gradients de tension interfaciale; l'interface aura alors tendance à onduler et à produire de petites gouttes. Ce phénomène est favorisé par l'agitation. Les conditions d'instabilité sont les suivantes (Gouda et Joos, 1975): la tension interfaciale à l'équilibre doit être très faible, le tensioactif doit être présent dans la phase dispersée, mais plutôt soluble dans la phase continue, et la viscosité de la phase dispersée doit être très supérieure à celle de la phase continue. Cette instabilité interfaciale s'avère primordiale pour l'émulsification d'une huile très visqueuse en phase aqueuse.

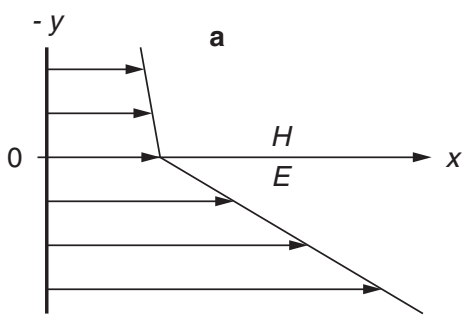

b
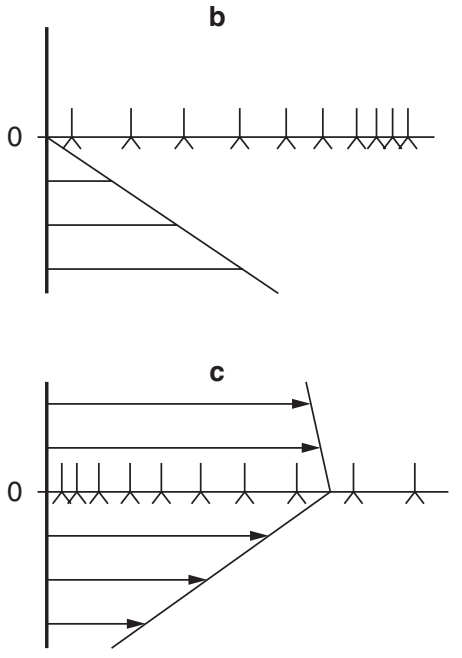

Figure 8

Gradients de tension interfaciale et écoulement près d'une interface huile/eau; (a) pas de tensioactif; (b) le gradient de vitesse provoque le gradient de tension interfaciale; (c) le gradient de tension interfaciale provoque l'écoulement (effet Marangoni) (d'après Walstra, 1997).

Interfacial tension gradients and flow close to an oil/water interface; (a) no surfactant; (b) the velocity gradient causes the interfacial tension gradient; $(c)$ the interfacial tension gradient causes flow (Marangoni effect) (from Walstra, 1997).

\subsection{Prévention de la coalescence}

La recoalescence de deux gouttelettes juste après leur formation pendant le processus d'émulsification a été récemment clairement mise en évidence (Taisne et al., 1996). La taille des gouttelettes est donc le résultat de deux processus : la rupture et la coalescence, et plus la concentration en tensioactif est élevée, plus la vitesse de coalescence est lente. On suppose souvent que la prévention de la coalescence est due à la 
répulsion colloïdale de la couche de tensioactif adsorbée. Cependant, durant l'émulsification, la rencontre mutuelle de deux gouttes fraîchement formées implique la mise en jeu d'une grande quantité d'énergie cinétique, et il est possible de calculer que l'énergie de répulsion, due par exemple aux forces électrostatiques, est en général trop faible pour empêcher le rapprochement des gouttelettes (Taisne et al., 1996). D'un autre côté, l'énergie libre impliquée dans la création des gradients de tension interfaciale devrait être suffisante pour arrêter le mouvement tangentiel de l'interface des gouttelettes qui se rapprochent, ralentissant de ce fait le drainage du film entre les gouttelettes.

On voit qu'il est difficile de faire la part de l'importance relative des phénomènes mis en jeu et il est probable que les processus réels impliqués pendant la formation de l'émulsion sont encore plus compliqués que ce que nous venons de décrire. En particulier, il ne faut pas oublier le rôle de l'effet Gibbs-Marangoni (fig. 9). Pendant l'émulsification, les gouttelettes ne possèdent pas encore la quantité de tensioactif nécessaire à l'équilibre, et le processus d'adsorption continue. Cependant, la quantité de tensioactif est moindre là où le film est plus mince. Par conséquent, la tension interfaciale sera plus élevée à ce point précis et la surface va se déplacer dans cette direction. L'effet Marangoni entraîne alors

a

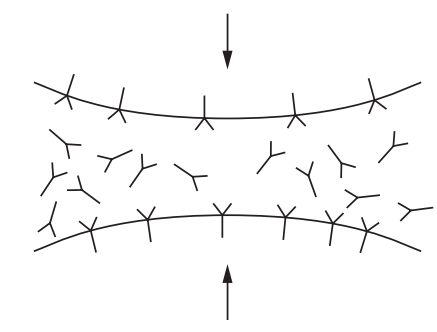

b

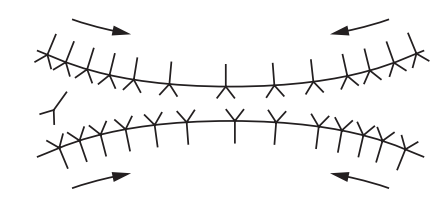

C

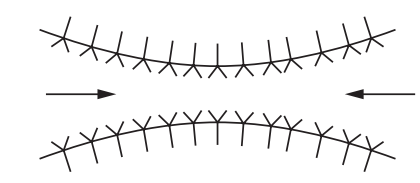

Figure 9

Schéma de l'effet Gibbs-Marangoni sur deux gouttelettes qui se rapprochent pendant l'émulsification (d'après Walstra, 1997).

Gibbs-Marangoni effect between two approaching droplets during emulsification (from Walstra, 1997). l'écoulement du liquide dans le film également vers le centre du film, ce qui contribue à tenir les gouttes éloignées. On aboutit ainsi à un mécanisme d'autostabilisation, à condition toutefois que l'élasticité de Gibbs $E_{f}$ du film soit suffisamment élevée. $E_{f}$ dépend essentiellement de la concentration en tensioactif et de l'épaisseur du film, et peut également dépendre du type de tensioactif.

\subsection{Type de l'émulsion}

La règle de Bancroft dit que la phase continue est celle où le tensioactif est le plus soluble. La figure 9 permet en effet d'expliquer ce phénomène. Si le tensioactif se trouvait dans les gouttelettes, il n'y aurait jamais de gradient de tension interfaciale dû à un manque de tensioactif au milieu du film. Par conséquent, on n'observerait pas d'effet GibbsMarangoni, et les gouttes coalesceraient au fur et à mesure de l'émulsification. Il est important de noter qu'il existe des exceptions à la règle de Bancroft. Elle reste cependant très utilisée comme base de prédiction (Schramm, 1992).

\section{FORMATION ET COALESCENCE DES GOUTTELETTES}

\subsection{Rupture des gouttelettes}

Le temps nécessaire à la rupture des gouttelettes est certainement du même ordre de grandeur que le temps de déformation. Théoriquement, l'émulsification devrait être réalisée en quelques secondes. Or, dans la pratique, l'ordre de grandeur est plutôt de quelques minutes.

En fait, on peut expliquer cet écart par le fait que techniquement, les machines les plus aptes à casser les très petites gouttes ne sont pas adaptées à la rupture des grosses gouttes en début d'émulsification et sont peu performantes. Par conséquent, en matière de fabrication d'émulsion, les meilleurs résultats sont obtenus en préémulsifiant le mélange dans un agitateur avant d'utiliser des machines comme les moulins à colloïdes ou les homogénéisateurs, qui permettent d'obtenir de très petites gouttes avec une distribution de tailles relativement serrée. Correctement employés, ces appareils peuvent donner des gouttes de 0,25 à $10 \mu \mathrm{m}$ en moins de 0,1 ms (Walstra, 1983). L'utilisation de machines moins performantes, comme les agitateurs, mettent en jeu des processus beaucoup plus lents, en particulier dans les dernières étapes de l'émulsification (formation des plus petites gouttes).

Les principaux facteurs qui affectent le temps d'émulsification sont la quantité et le type de tensioactif, et la vitesse d'agitation. L'effet de la fraction volumique n'est pas très clair; il est probablement faible si l'on maintient la concentration en tensioactif constante dans la phase continue. 
Le cassage des gouttelettes en cours d'émulsification est souvent traité comme une réaction du premier ordre: $\mathrm{d} N / \mathrm{d} t=K N$, avec $K$ constante de vitesse $\left(\mathrm{s}^{-1}\right)$ et $N$ nombre de gouttes. Par conséquent, on peut aboutir à une relation donnant la taille moyenne des gouttelettes en fonction du temps:

$$
\bar{d}=\frac{\overline{d_{\infty}}}{1-\exp (-K t)}
$$

avec $\mathrm{d}_{\infty}$ le diamètre moyen final. Cependant, la plupart du temps, cette équation ne correspond pas aux résultats expérimentaux, sans doute parce qu'il existe un grand nombre de réactions intermédiaires impliquées dans le processus global et qui dépendent toutes plus ou moins de la taille des gouttelettes. Il faut bien avoir à l'esprit que dans les appareils utilisés, quels qu'ils soient, la densité d'énergie varie fortement localement, et qu'il peut se passer beaucoup de temps avant que le mélange ne passe dans la zone de l'appareil la plus favorable au cassage des gouttelettes. Dans certains cas, il peut même s'avérer nécessaire d'augmenter le nombre de passages dans l'appareil.

De nombreux facteurs sont susceptibles de modifier la vitesse d'émulsification globale, et il ne suffit pas d'augmenter le temps ou la vitesse d'agitation pour obtenir la granulométrie désirée. Quelquefois, le fait d'agiter plus longtemps va provoquer une augmentation de la température locale et favoriser la formation de petites gouttelettes par abaissement de la viscosité de la phase dispersée. Dans d'autres cas, on va obtenir une augmentation de la taille finale de l'émulsion après être passé par un minimum, tout simplement par suite d'une décomposition des tensioactifs qui ne supportent pas l'élévation de température. La prédiction de la cinétique d'émulsification reste donc très difficile.

\subsection{Coalescence en cours d'émulsification}

Certains auteurs pensent que la valeur finale de la taille des gouttelettes obtenues est le résultat d'un équilibre entre la coalescence et la rupture (Gopal, 1968). La cinétique d'émulsification est alors donnée par $\mathrm{d} N / \mathrm{d} t=K_{1} N-K_{2} N^{2}, K_{2}$ étant la constante de vitesse de la coalescence, et $N_{\infty}=K_{1} / K_{2}$ (nombre de gouttes final).

D'autres auteurs pensent que la coalescence intervient probablement dans le processus d'émulsification, mais qu'elle concerne surtout les gouttelettes qui viennent juste de se former et ne possèdent pas encore de couche d'adsorption à l'équilibre (Walstra, 1983).

À l'heure actuelle, l'importance quantitative de la coalescence dans le processus d'émulsification est inconnue. Tout ce qu'il est possible de dire, c'est qu'elle doit être très variable et doit permettre d'expliquer en partie pourquoi l'on obtient des granulométries différentes suivant le type de tensioactif utilisé. Cependant, les travaux récents de Taisne et al. (1997) et Taisne (1997) ont permis de vérifier, dans le cas d'un homogénéisateur haute pression, qu'il existait bien deux régimes d'émulsification liés à la disponibilité du tensioactif. Aux faibles concentrations, les gouttes recoalescent dans la valve et à la sortie de la valve de l'homogénéisateur; la taille finale reflète alors l'étendue de la recoalescence. Aux concentrations élevées, les gouttes ne recoalescent pas; la distribution de tailles finale reflète l'efficacité de la fragmentation.

\section{PRINCIPALES CONCLUSIONS SUR LA FORMATION DES ÉMULSIONS}

La rupture des gouttes en écoulement laminaire et turbulent est un phénomène désormais relativement bien compris. Reste à mieux cerner d'un point de vue quantitatif l'effet de la viscosité de la phase dispersée en écoulement turbulent. On comprend également bien le rôle du tensioactif, mais ses effets sont difficiles à prédire quantitativement, surtout en ce qui concerne les polymères. Le rôle de l'élasticité de Gibbs est également difficile à appréhender.

Le tableau 5 reprend les principales relations concernant l'ordre de grandeur du diamètre des gouttes et des temps caractéristiques en fonction des régimes d'émulsification.

\section{TABLEAU 5}

Diamètre des gouttes et temps caractéristiques en fonction des divers régimes d'émulsification (d'après Walstra, 1997)

Droplet diameter and characteristic time scales as a function of different emulsification patterns (from Walstra, 1997)

\begin{tabular}{l|c|c|c}
\hline $\begin{array}{c}\text { Régime } \\
\text { d'écoulement }\end{array}$ & $\begin{array}{c}\text { Laminaire } \\
\text { Forces } \\
\text { de cisaillement }\end{array}$ & $\begin{array}{c}\text { Turbulent } \\
\text { Forces } \\
\text { de cisaillement }\end{array}$ & $\begin{array}{c}\text { Turbulent } \\
\text { Forces } \\
\text { d'inertie }\end{array}$ \\
\hline $\operatorname{Re}$ (écoulement) & $<1000$ & $>2000$ & $>2000$ \\
\hline $\operatorname{Re}_{g}$ (gouttelette) & $<1$ & $\frac{\gamma}{\gamma}$ & $\frac{\gamma^{* / 5}}{\varepsilon^{1 / 2} \eta_{c}^{1 / 2}}$ \\
\hline Diamètre $d$ & $\frac{2 \gamma \mathrm{Ca}_{c r}}{\eta_{c} G}$ & $\frac{\eta_{d}}{5 \varepsilon^{1 / 2} \eta_{c}^{1 / 2}}$ & $\frac{\eta_{d}}{5 \varepsilon^{2 / 3} d^{2 / 3} \rho^{1 / 3}}$ \\
\hline$\tau_{\text {ads }}$ & $\frac{\eta_{d} G}{\eta_{c} G}$ & $\frac{10 \Gamma \eta_{c}^{1 / 2}}{d m_{c} \varepsilon^{1 / 2}}$ & $\frac{10 \Gamma \eta_{c}^{1 / 2}}{d m_{c} \varepsilon^{1 / 2}}$ \\
\hline$\tau_{\text {col }}$ & $\frac{\pi}{8 \phi G}$ & $\frac{\pi \eta_{c}^{1 / 2}}{8 \phi \varepsilon^{1 / 2}}$ & $\frac{d^{2 / 3} \rho^{1 / 3}}{8 \phi \varepsilon^{1 / 3}}$ \\
\hline
\end{tabular}

* Pour $d>\eta_{c}^{2} / \gamma \rho$

** Uniquement pour $\eta_{d} \gg \eta_{c}$

Le tableau 6 rassemble les mécanismes de dispersion, la taille moyenne des gouttes, les densités d'énergie et de puissance, les temps de séjour et les diverses variables impliquées dans les équipements d'émulsification en continu. 
La figure 10 illustre le schéma de fonctionnement des types d'appareils considérés.

Pour les équipements d'émulsification en continu, les auteurs préfèrent utiliser la notion de densité d'énergie $E_{v}$, qui est l'énergie dissipée dans un volume $V: E_{v}=\varepsilon \cdot t_{\text {séjour }}$ $=P / Q$, où $t_{\text {séjour }}$ est le temps de séjour moyen dans la zone de dispersion, $P$ la puissance appliquée et $Q$ le débit volumique.
Dans le cas d'un cassage continu des gouttelettes, le diamètre de Sauter moyen $\left(d_{32}\right)$ est fonction de $E_{v}$ (tableau 6 ). Les systèmes de dispersion haute pression, pour lesquels la densité d'énergie est déterminée uniquement par la pression, sont particulièrement adaptés à la production d'émulsions de très petite taille pour toute viscosité. À densités d'énergie égales, les systèmes à jet sont plus efficaces car le temps de séjour

TABLEAU 6

Procédés d'émulsification en continu (d'après Schubert et Stang, 1997)

Continuous emulsification processes (from Schubert and Stang, 1997)

\begin{tabular}{|c|c|c|c|c|c|}
\hline Appareil & Membrane & $\begin{array}{r}\text { Ro } \\
\text { Lisse (moulin) }\end{array}$ & stator & $\begin{array}{l}\text { Homogénéisateur } \\
\text { HP }\end{array}$ & $\begin{array}{l}\text { Injection } \\
\quad(\text { jet })\end{array}$ \\
\hline $\begin{array}{l}\text { Mécanisme } \\
\text { de dispersion }\end{array}$ & $\begin{array}{l}\text { formation de gouttes } \\
\text { dans les capillaires }\end{array}$ & $\begin{array}{l}\text { laminaire } \\
\text { cisaillement simple } \\
\text { + élongationnel }\end{array}$ & $\begin{array}{l}\text { turbulent } \\
\text { cisaillement } \\
\text { et forces d'inertie }\end{array}$ & \multicolumn{2}{|c|}{$\begin{array}{l}\text { turbulent, forces de cisaillement et d'inertie } \\
\text { cisaillement élongationnel } \\
\text { cavitation }\end{array}$} \\
\hline Taille des gouttes & $x_{\text {moy }}=C_{1} \phi E_{v}^{-1}+C_{2}$ & \multicolumn{2}{|c|}{$x_{32}=C E_{v}^{-b}$} & $\begin{array}{l}\left(E_{v}=P / Q=\Delta P\right) \\
b \approx 0,6(0,4-0,9)\end{array}$ & \\
\hline$E_{v}\left(\mathrm{~J} / \mathrm{m}^{3}\right)$ & $<10^{3}-10^{6}$ & $10^{3}-10^{5}$ & $10^{5}-10^{8}$ & \multicolumn{2}{|c|}{$10^{6}-10^{8}$} \\
\hline Temps de séjour (s) & 1 & \multicolumn{2}{|c|}{$0,1-1$} & $10^{-3}$ & $10^{-4}$ \\
\hline$\varepsilon\left(\mathrm{W} / \mathrm{m}^{3}\right)$ & $<10^{3}-10^{6}$ & $10^{3}-10^{6}$ & $10^{5}-10^{9}$ & $10^{9}-10^{11}$ & $10^{10}-10^{12}$ \\
\hline Variables de contrôle & $\begin{array}{l}\qquad \eta_{d} \\
\text { design du capillaire, } \\
\text { mouillabilité }\end{array}$ & $\begin{array}{l}\eta_{d} / \eta_{c} \\
(0,1-1)\end{array}$ & \multicolumn{3}{|c|}{$\begin{array}{l}\text { cinétiques d'adsorption } \\
\text { viscosité de la phase dispersée }\end{array}$} \\
\hline $\begin{array}{l}\text { Variables } \\
\text { de peu d'influence }\end{array}$ & & & \multicolumn{3}{|c|}{$\begin{array}{l}\text { teneur en huile }(\phi) \text { viscosité de la phase continue }\left(\eta_{c}\right) \\
\text { design des « dents » }\end{array}$} \\
\hline
\end{tabular}

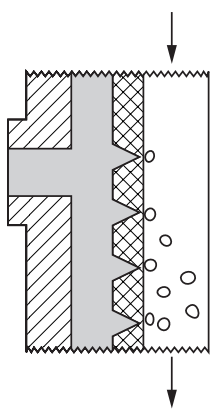

a

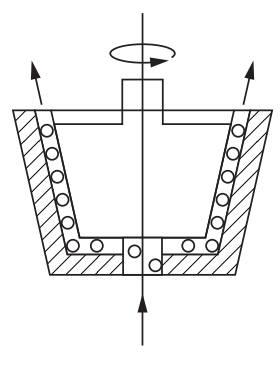

b

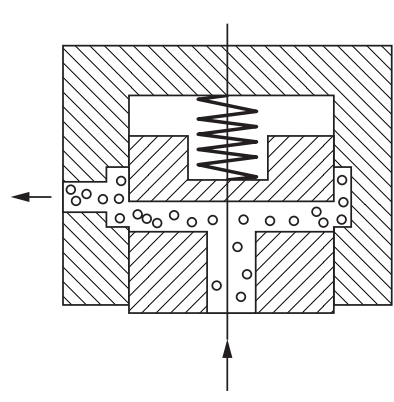

C

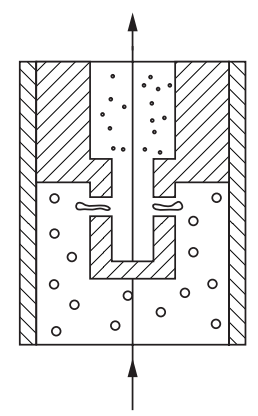

d

Figure 10

Équipements d'émulsification continus (d'après Schubert et Stang, 1997) ; (a) émulsification par membrane ; (b) rotor-stator ; (c) homogénéisateur haute pression ; (d) système de dispersion par jet.

Continuous emulsification equipment (from Schubert and Stang, 1997); (a) membrane emulsification; (b) rotor-stator system; (c) highpressure homogenizer; (d) jet-dispersing system. 
moyen est plus court, donc la densité de puissance plus élevée. De même, le volume de la zone de dispersion est généralement beaucoup plus petit dans les équipements haute pression par rapport aux systèmes du type rotor-stator. Pour une même densité d'énergie et un même débit volumique, les densités de puissance sont beaucoup plus grandes. Notons enfin que les forces de cavitation et élongationnelles rencontrées dans les systèmes haute pression améliorent et aident la production de gouttes plus petites pour une même densité d'énergie.

D'un point de vue purement pratique, il est particulièrement intéressant de retenir les points suivants (Walstra, 1983):

- le choix de l'appareil d'émulsification ne dépend pas seulement de la granulométrie recherchée. Cependant, en règle générale, pour les viscosités élevées de phase continue, on préférera utiliser un moulin à colloïdes. Si l'on recherche une taille moyenne de gouttelettes très petite, on choisira un homogénéisateur haute pression. N'oublions pas non plus qu'une préémulsification dans un simple agitateur peut améliorer considérablement les performances de ce type d'appareil;

- l'effet d'échelle est très important, surtout en ce qui concerne la quantité d'énergie nécessaire. Dans le cas du moulin à colloïdes, on s'attend à ce que le diamètre moyen obtenu soit proportionnel à $P^{-1}$; il peut y avoir cependant de grandes différences entre l'énergie totale fournie et l'énergie réellement utilisée, surtout pour les petits appareils;

- la durée d'agitation (pour les opérations en batch) ou le nombre de passes (pour les opérations continues) sont des paramètres très importants. En général, ils entraînent une diminution de la taille moyenne et de la largeur de la distribution;

- les propriétés rhéologiques de chacune des phases interviennent également. La viscosité de la phase continue affecte le nombre de Reynolds, donc le type d'écoulement, et détermine le mécanisme de rupture des gouttelettes (écoulement laminaire ou turbulent, avec forces visqueuses ou d'inertie). En écoulement de cisaillement simple, la rupture dépend essentiellement du rapport des viscosités $q$. En écoulement élongationnel, $q$ a peu d'effet. Dans les deux cas, la taille moyenne diminue quand la viscosité augmente. En écoulement turbulent, les viscosités ont peu d'effet. Les équations sont beaucoup plus compliquées avec les fluides non newtoniens;

- la concentration et la nature du ou des tensioactifs sont d'une importance primordiale pour la stabilité de l'émulsion, mais elles affectent également la taille des gouttelettes. Plus la densité d'énergie est élevée, moins l'effet du tensioactif sur la taille moyenne est marqué. Pour la plupart des anioniques, l'augmentation de la concentration entraîne une taille moyenne plus petite, jusqu'à une valeur plateau (vers la cmc, concentration micellaire critique). Pour beaucoup de non-ioniques, plus l'on utilise de tensioactif, plus l'énergie nécessaire à l'obtention d'une certaine taille est faible, même au-delà de la cmc. Pour les polymères, on observe également une diminution de la taille lorsqu'on augmente la concentration, avec quelquefois un minimum, et non plus une valeur plateau;

- la fraction volumique $\phi$ est le ratio volume dispersé sur volume total. La plupart du temps, la taille moyenne augmente avec $\phi$ (autres conditions constantes), mais l'effet est peu prononcé;

- la méthode de préparation reste bien sûr un paramètre clé de l'émulsification. L'ordre d'addition des ingrédients est souvent primordial. En général, il est recommandé d'ajouter la phase dispersée au fur et à mesure de l'émulsification (goutte à goutte par exemple). On obtient également de bons résultats avec une préémulsification;

- la température intervient à de nombreux niveaux dans le processus de fabrication d'émulsion. Elle affecte notamment les viscosités des deux phases, donc le type d'écoulement, et également la solubilité des tensioactifs dans chacune des phases.

\section{CAS PARTICULIER DES ÉMULSIONS PÉTROLIÈRES}

\subsection{Le problème des émulsions pétrolières}

Dans l'industrie qui nous intéresse, c'est-à-dire l'industrie pétrolière, l'eau et le pétrole sont produits simultanément et sont principalement non miscibles. En effet, les solubilités des hydrocarbures dans l'eau varient considérablement mais restent très faibles (Yaws et al., 1990), de 0,0022 ppm pour le tetradécane à 1,76 ppm pour le benzène dans les conditions standard. Par conséquent, ces deux liquides coexistent, ou bien sous forme de couches stratifiées distinctes, ou bien sous forme d'émulsions ou de dispersions d'une phase dans l'autre.

Bien que d'un point de vue purement théorique, toute dispersion d'un liquide dans un autre soit une émulsion, quelle que soit la taille des gouttelettes formées, l'industrie pétrolière distingue trois types de mélanges en fonction des mécanismes de séparation des phases impliquées (Davies et al., 1996):

- les dispersions primaires sont des mélanges eau/huile ou huile/eau thermodynamiquement instables et qui se séparent sous l'action de la gravité. La vitesse de séparation est directement proportionnelle à la taille des gouttelettes (diamètre moyen $>60 \mu \mathrm{m}$ ) ;

- les dispersions secondaires sont également des mélanges instables, mais le temps de décantation des gouttelettes est supérieur au temps de séjour moyen des effluents dans les équipements conventionnels de séparation (diamètre moyen de 30 à $60 \mu \mathrm{m})$; 
- les émulsions sont des dispersions très fines (diamètre moyen $<30 \mu \mathrm{m}$ ) qui sont considérées comme stables dans l'échelle de temps du procédé, bien que thermodynamiquement instables.

En fait, si le temps de demi-vie des dispersions primaires n'est que de 30 secondes en moyenne, celui des dispersions secondaires est de l'ordre de quelques heures, alors que celui des émulsions est de l'ordre de quelques semaines.

Pour des raisons pratiques, nous conserverons le terme générique d'émulsion pour désigner tous ces types de mélanges.

Comme nous l'avons mentionné en introduction, les émulsions pétrolières se trouvent à tous les niveaux de la chaîne: fluides de forage, production, raffinerie, sans oublier le transport. En production plus particulièrement, les émulsions indésirables, qui posent de gros problèmes, sont les émulsions formées en tête de puits. À ce niveau, toutes les conditions sont en effet réunies pour former des émulsions qui peuvent s'avérer très stables: présence des deux phases eau et pétrole, zones d'agitation très forte au niveau de la choke valve (duse de sortie), présence d'agents tensioactifs (bactéricides, inhibiteurs de corrosion, etc.) et de particules très fines (sables, sulfures de fer, argiles, etc.) qui peuvent stabiliser l'émulsion.

Sur le champ de production, les émulsions eau dans huile (e/h) sont appelées émulsions directes (car ce sont les plus courantes) et les émulsions huile dans eau (h/e) sont dites inverses. Cependant, il faut garder à l'esprit que l'on peut se trouver en présence de systèmes beaucoup plus complexes, comme des émulsions multiples e/h/e ou h/e/h.

Les émulsions les plus fréquemment rencontrées sont donc du type e/h et sont dites directes. La phase aqueuse dispersée est généralement appelée eau et sédiments (sediment and water, $\mathrm{S} \& \mathrm{~W}$ ) et la phase continue est le pétrole brut. La phase $\mathrm{S} \& \mathrm{~W}$ est essentiellement constituée d'eau salée, mais l'on y trouve aussi des solides comme du sable, de la boue, des produits de corrosion, des dépôts de tartre, des précipités solides, etc.

\subsection{Facteurs affectant l'émulsification lors de la production}

L'effet des techniques classiques de production peut être rapidement résumé: au moins $90 \%$ des pratiques actuelles peuvent générer ou aggraver les problèmes d'émulsion. Tout d'abord, les produits chimiques utilisés lors de la fracturation de la formation, les workovers, les stimulations de puits, les inhibiteurs de corrosion, etc. (Gidley et Hanson, 1974), puis les différentes techniques de récupération assistée (fireflooding, injection de polymères, injection de $\mathrm{CO}_{2}$ ou de vapeur) peuvent en effet causer de graves problèmes d'émulsion. Enfin, une cause importante de la formation d'émulsions en production reste l'agitation provoquée par les pertes de charge brutales occasionnées par les choke valves ou les pompes.

Les produits susceptibles de favoriser la formation des émulsions e/h sont particulièrement nombreux (Svetgoff, 1989):

- composés tensioactifs naturellement contenus dans le pétrole brut: asphaltènes, résines, acides naphténiques, acides carboxyliques, composés soufrés, phénols, crésols et tous les autres surfactifs de haut poids moléculaire;

- solides finement divisés: sable, argiles, fines de formation, débris de coquillages, silts, gilsonite, boues de forage, fluides de workover, dépôts minéraux, composés de corrosion (FeS, rouille), paraffines cristallisées, asphaltènes précipités et résines;

- produits chimiques ajoutés: inhibiteurs de corrosion, biocides, détergents, agents mouillants, produits de stimulation.

Peu d'études ont été publiées sur l'influence des équipements mécaniques sur la formation des émulsions (Shea, 1939; Flanigan et al., 1992) alors que l'effet des produits chimiques est bien documenté. On sait bien sûr que les pompes centrifuges sont à éviter, et qu'on leur préférera des pompes générant de faibles cisaillements ou pertes de charge.

\subsection{Prévention de l'émulsification}

Les avis sont très partagés quant à la présence d'émulsions dès la formation, mais il est clair pour tout le monde que si les effluents sont stratifiés en fond de puits, il y a formation d'émulsions lors de la remontée dans le puits ou dans les équipements de surface.

Prévenir la formation de ces émulsions, c'est avant tout agir à deux niveaux. Il faut d'abord éliminer ou restreindre les zones de turbulence et de fort cisaillement. Il faut ensuite séparer l'eau de l'huile le plus tôt possible dans les équipements de surface.

Considérons les principales sources d'émulsification suivant le type de puits (Manning et Thompson, 1995):

- dans les puits éruptifs, une agitation considérable est fournie par le gaz qui s'échappe lorsque la pression diminue. Ce gaz provoque également des turbulences importantes lorsqu'il passe à travers toutes sortes de restrictions (perforations, filtres, raccords, duses, étranglements, coudes à angle droit, etc.). La turbulence peut être limitée, mais pas empêchée, en installant des duses plus larges ou en utilisant des choke valves en cascade. Une duse en fond de puits provoque généralement moins de problèmes d'émulsification, et ce pour différentes raisons: il y a moins de perte de charge au niveau d'une duse en fond de puits, les températures sont plus élevées qu'en surface et l'écoulement est peu turbulent en aval de la duse; 
- dans les puits activés par gas lift, l'émulsification se produit essentiellement à deux niveaux : au point d'injection du gaz et en tête de puits. Quand il s'agit de gas lift intermittent, l'émulsion se crée surtout en tête de puits ou dans les équipements de surface. En gas lift continu, l'émulsion est surtout formée en fond de puits aux points d'injection du gaz;

- dans les puits pompés, les principales causes d'émulsification sont dues aux pompes et aux conduites: valves d'étanchéité, clapets de refoulement, pistons et autres composants des pompes; zones de turbulence créées par le passage du gaz dans la pompe ou par cognement des tiges.

Toutes ces sources de turbulence peuvent être minimisées en utilisant une pompe avec des clapets d'étanchéité et de refoulement légèrement surdimensionnés, ainsi qu'une vitesse et une longueur de course adaptées. Sur les puits producteurs de gaz, l'installation d'un piège à gaz réduit en général la turbulence.

Enfin, en surface, les principales causes de turbulence dans les manifolds et les lignes de collecte sont les pompes, les valves et les raccords de conduites dans lesquels la direction de l'écoulement change brusquement.

\subsection{Application des théories sur l'émulsification à la production de pétrole}

Comment appliquer ces théories sur la formation des émulsions au cas particulier de la production de pétrole brut? Il est clair que la tâche n'est pas facile:

- on est en présence de plusieurs sources potentielles d'émulsification (pompes, vannes, duses, accidents de conduites, etc.) que l'on ne peut pas simplement comparer à un moulin à colloïdes ou à un homogénéisateur;

- les fractions volumiques e/h couvrent une très large gamme (de quelques pour-cent à plus de $90 \%$ d'eau);

- on peut rencontrer aussi bien des émulsions e/h que $\mathrm{h} / \mathrm{e}$, voire des émulsions multiples e/h/e ou h/e/h;

- on se retrouve souvent en écoulement triphasique (eauhuile-gaz);

- si la phase aqueuse peut être relativement facilement reconstituée, le pétrole brut est un produit très complexe comprenant des milliers de molécules différentes et qu'il est difficile de représenter par une huile de mêmes viscosité, densité et tension interfaciale.

Très peu de publications ont paru sur l'influence des différents équipements sur la formation de dispersions ou d'émulsions eau-pétrole brut. Récemment, deux études intéressantes ont été publiées sur la formation de dispersions eau-brut dans des conditions de température et de pression plus proches de la réalité que ce que l'on réalise au laboratoire sur les huiles « mortes » (c'est-à-dire ayant perdu leur gaz). Ronningsen et Urdahl (1995) ont étudié la stabilité d'émulsions de brut de mer du Nord et d'eau reconstituées à travers une choke valve en présence de méthane. Ils ont mis clairement en évidence l'influence de la pression et du gaz dissous sur la stabilité des émulsions formées et observées à température et pression ambiantes. Les expériences de laboratoire réalisées avec les huiles mortes aux conditions ambiantes (pression atmosphérique, température autour de $20^{\circ} \mathrm{C}$ ) donnent des résultats comparables en tendance mais beaucoup plus pessimistes en ce qui concerne la stabilité (donc la taille des gouttes formées). Davies et al. (1996) ont également étudié la stabilité de dispersions eau-brut reconstituées en température et en pression à travers une choke valve. Contrairement à l'étude précédente, la stabilité des dispersions formées a été observée dans une «cellule séparateur» maintenue en pression. Différents paramètres ont été étudiés: type de brut, rapport eau/huile, $\Delta P$ à travers la choke valve, conditions opératoires $(T, P)$ effet des paraffines et des asphaltènes et effet de tensioactifs désémulsionnants. La principale conclusion de ce travail a été la mise en évidence de l'existence d'un water cut (rapport eau/huile) critique, en deçà duquel la séparation devient très difficile, et de l'influence importante des paraffines et des asphaltènes.

En ce qui concerne plus précisément l'étude du cassage de gouttelettes dispersées soumises à différentes sources d'énergie, on conçoit très facilement la très grande difficulté que posent les dispersions eau dans pétrole brut, dont les principales caractéristiques sont d'être opaques, souvent concentrées et non conductrices. La détermination de la taille de ces dispersions ne peut donc pas se faire par les techniques conventionnelles. C'est pourquoi, pour ce type de dispersions, la détermination de la taille des gouttelettes se fait généralement indirectement via les études de stabilité. Le problème est beaucoup plus simple pour les dispersions pétrole brut dans eau, que l'on va observer en règle générale pour des water cuts élevés (Davies et al., 1996), mais les résultats expérimentaux de ce type sont très rares dans la littérature.

Très récemment, van der Zande et van den Broek (1998) ont publié une étude sur le cassage de gouttelettes d'huile dans l'eau, représentatif de ce qui se passe en production d'huile lors du passage de l'eau et du brut dans le tubing et la choke valve. Les huiles choisies pour ces expériences sont des huiles minérales de viscosités différentes (Shell Vitrea 9 et 46). Deux montages expérimentaux ont été réalisés pour analyser l'effet d'un écoulement en conduite et d'une choke valve sur la distribution en taille des gouttelettes d'huile. Pour l'écoulement en conduite, les auteurs ont utilisé un tube droit de $2 \mathrm{~m}$ ( $15 \mathrm{~mm}$ de diamètre interne) et un tube hélicoïdal de $16 \mathrm{~m}$ avec un rayon de courbure de $0,5 \mathrm{~m}$ et de même diamètre intérieur. Pour simuler la choke valve, des orifices calibrés ont été utilisés, de diamètre de 4 à $0,5 \mathrm{~mm}$, pour un diamètre de tube de $4,5 \mathrm{~mm}$. La distribution en taille des gouttelettes a été mesurée avec un granulomètre laser 
Malvern 3600 E. La concentration volumique d'huile dans l'eau est de l'ordre de $0,5 \%$.

En ce qui concerne l'écoulement turbulent en conduite, les auteurs ont considéré trois relations permettant de prédire le diamètre maximal des gouttes: tout d'abord l'équation classique de Hinze (1955) (équation (34): $d_{\max }=C \theta^{2 / 5} \gamma^{3 / 5} \rho^{-3 / 5}$ $u^{6 / 5}$, avec $\left.C=0,725\right)$, puis la relation empirique de Sleicher (1962) qui prend en compte la viscosité de la phase dispersée:

$$
\frac{d_{\max } \rho_{c} u^{2}}{\gamma} \sqrt{\frac{\eta_{c} u}{\gamma}}=38\left[1+0,7\left(\frac{\eta_{d} u}{\gamma}\right)^{0,7}\right]
$$

( $u$ étant la vitesse moyenne dans la conduite) et, pour terminer, la relation de Karabelas (1978), qui introduit la notion de dissipation locale d'énergie dans la couche logarithmique $\varepsilon_{l}$ à la place de $\varepsilon$ (densité de puissance moyenne) et aboutit à la relation:

$$
\frac{d_{95}}{D_{c}}=4,0 \mathrm{We}^{-0,6}
$$

avec We $=\rho_{c} u^{2} D_{c} / \gamma, D_{c}$ étant le diamètre de la conduite et $d_{95}$ le diamètre de gouttelettes en dessous duquel $95 \%$ de la population en volume est représentée.

Les relations de Sleicher et Karabelas sont semiempiriques et sont fondées sur l'hypothèse que la rupture des gouttelettes en écoulement en conduite turbulent se fait essentiellement à la paroi.

Les résultats expérimentaux de van der Zande et van den Broek montrent que la taille des gouttelettes diminue lorsqu'on augmente le débit, et augmente avec la viscosité de l'huile dispersée. Cependant, aucune des trois relations n'est capable de prédire la taille maximale des gouttelettes formées lors de l'écoulement, même avec un système aussi simple que celui choisi par les auteurs (système liquide/liquide très dilué).

Pour la simulation de l'écoulement à travers une choke valve, le système a été simplement modélisé par l'utilisation d'un orifice calibré dans un tube circulaire. L'endroit où la pression minimale est atteinte est appelé vena contracta. La perte de charge maximale est donnée par l'équation suivante:

$$
\Delta p_{\max }=\frac{1}{C_{o}^{2}} \frac{1}{2} \rho_{c} u^{2}\left(\frac{1}{\beta^{4}}-1\right)
$$

où $C_{o}$ est le coefficient d'orifice, $\beta$ le rapport entre le diamètre de l'orifice $D_{o}$ et le diamètre de la conduite $D_{c}$, et $u$ la vitesse moyenne dans la conduite. La force de déformation imposée aux gouttelettes est donc proportionnelle à cette perte de charge maximale (Percy et Sleicher, 1983):

$$
F_{d}=\Delta p_{\max } d / D_{o}
$$

et le diamètre maximal des gouttes est donné par :

$$
d_{\max }=C\left(\frac{D_{o} \gamma}{\Delta p_{\max }}\right)^{1 / 2}
$$

avec $C=3,1$ dans le cas des expériences menées par Percy et Sleicher.

Van der Zande et van den Broek ont comparé leurs résultats expérimentaux au diamètre maximal calculé selon la relation (43). Ils concluent que cette relation permet seulement une description approximative de la rupture des gouttelettes à travers un orifice. Elle a tendance à sous-estimer la taille de gouttelettes obtenues avec de grandes pertes de charge et de petits orifices. Elle ne prend pas non plus en compte la viscosité de la phase dispersée, alors que van der Zande et van den Broek ont observé une augmentation de la taille avec la viscosité de l'huile.

L'ensemble de ces travaux confirme la complexité théorique de la description de la rupture de gouttelettes dans les milieux dilués. Qu'en est-il des mélanges plus concentrés, qui, répétons-le, constituent la majeure partie des systèmes rencontrés en production pétrolière?

Le moins que l'on puisse dire est que les études publiées dans la littérature à ce sujet sont rares. On trouve un grand nombre de travaux sur la distribution de tailles de gouttes dans les dispersions liquide/liquide turbulentes en cuves agitées, et la majorité utilise la théorie de Hinze-Kolmogorov pour prédire le diamètre stable maximal. Rappelons que cette théorie postule que dans le spectre initial de turbulence, seuls les tourbillons dont la taille est inférieure au diamètre de la goutte peuvent causer la rupture de cette goutte. Les modèles reposant sur cette théorie aboutissent à une loi de puissance décroissante du diamètre maximal stable en fonction de la dissipation d'énergie turbulente moyenne (exposant -0,4) ou, sous une autre forme, en fonction de la vitesse de rotation de l'agitateur (exposant -1,2) (éq. (34)). Par conséquent, le diamètre moyen de Sauter de la distribution suit la même tendance. De nombreuses données expérimentales en cuves agitées peuvent être interprétées selon cette théorie, mais leur validité se limite au cas des dispersions diluées (quelques pour-cent). L'effet de la fraction de phase dispersée $\phi$ sur le diamètre moyen résultant a également été étudié et les résultats sont souvent présentés sous cette forme:

$$
d_{32} / X \approx\left(1+a \phi^{n}\right) \mathrm{We}^{-0,6}
$$

avec $X$ diamètre de l'agitateur et We nombre de Weber de l'agitateur. Par conséquent, si l'on applique la même puissance d'agitation, l'augmentation de la fraction volumique entraîne la formation de plus grosses gouttes dans la cuve agitée. Cette tendance a été observée pour des systèmes à forte ou faible coalescence (Lagisetty et al., 1986; Kumar et al., 1991). Le cas des fractions volumiques élevées a été très peu étudié et, la plupart du temps, il est très difficile de 
distinguer les effets dus à la coalescence et ceux relevant de la fraction volumique de phase dispersée.

Récemment, une étude très intéressante a été publiée (Desnoyer et al., 1998) sur la détermination expérimentale de la distribution de tailles de gouttes en cuves agitées de dispersions liquide/liquide fortement concentrées (jusqu'à 60\%). Les auteurs ont étudié deux types de dispersions eau dans huile se comportant différemment en termes de coalescence (coalescence rapide et coalescence lente). Ils ont développé une technique d'échantillonnage et de dilution manuelle très délicate pour pouvoir déterminer la distribution de tailles des gouttes par granulométrie laser. Les résultats de cette étude montrent que pour chaque fraction volumique étudiée, le diamètre moyen de la dispersion est une loi de puissance décroissante du nombre de Weber de l'agitateur avec un exposant de $-0,6$ pour les faibles concentrations. Mais il s'est avéré également que pour les deux types de système étudiés, cet exposant est une fonction décroissante de $\phi$. Ce résultat important met en évidence l'existence d'un mécanisme plus complexe de rupture des gouttes à $\phi$ élevée que ce que prédit la théorie. Les résultats montrent également l'existence d'une distribution de tailles de gouttes secondaires (gouttes satellites). Le rapport entre le diamètre moyen de la distribution primaire et celui de de la distribution secondaire n'est pas affecté par la fraction volumique de la phase dispersée, et les ordres de grandeur mesurés sont en bon accord avec les prédictions de Stone (1994).

\section{CONCLUSION}

La rupture des gouttes en conditions laminaires ou turbulentes est un phénomène relativement bien compris. Reste désormais tout le problème de la prédiction quantitative de la taille des gouttes en milieu concentré, qui nécessite la connaissance d'un grand nombre de données physicochimiques et hydrodymamiques:

- masse volumique, viscosité et tension interfaciale des phases en présence;

- énergie dissipée en fonction de la géométrie considérée et du régime d'écoulement: nous avons vu que cette notion est très importante; l'énergie volumique $E_{v}\left(\mathrm{~J} / \mathrm{m}^{3}\right)$ ou la densité de puissance $\varepsilon\left(\mathrm{W} / \mathrm{m}^{3}\right)$ peuvent être calculées, mais le problème reste de savoir si la dissipation d'énergie peut être considérée comme uniforme dans la zone de dispersion considérée;

- tensioactifs: nous avons vu que leur rôle va bien au-delà du simple abaissement de la tension interfaciale et de la détermination du type de l'émulsion; ils interviennent aux niveaux du mode de déformation et de la prévention de la recoalescence.

Par conséquent, pour des systèmes très concentrés où les éléments tensioactifs ainsi que les conditions hydrodynamiques sont difficilement identifiables, comme c'est le cas pour les émulsions pétrolières, il apparaît nécessaire de bien identifier les paramètres pertinents qui vont avoir un rôle essentiel dans les mécanismes de rupture et de coalescence des gouttelettes. L'acquisition de données expérimentales fiables sur des systèmes parfaitement contrôlés, à défaut d'être parfaitement connus, apparaît comme une priorité.

\section{REMERCIEMENTS}

Le travail présenté a été effectué dans le cadre de l'ARTEP (Association de recherche sur les techniques d'exploitation du pétrole) avec le soutien financier du Fonds de soutien aux hydrocarbures. Le sujet d'étude fait partie du projet ARTEP «Maîtrise des eaux en production: caractérisation des émulsions/dispersions » en collaboration Total-EEP-IFP. L'auteur remercie l'ARTEP de son autorisation de publication.

\section{RÉFÉRENCES}

Acrivos, A. (1974) Proc. Intern. Colloquium on Drops and Bubbles, Collins, D.J., Plesset, M.S. et Saffren, M.H. (éd.), Jet Propulsion Laboratory, Pasadena, Californie, 390 sq.

Ambruster, H. (1990) Untersuchungen zum kontinuierlichen Emulgierprozess, in Kolloidmühlen unter Berücksichtigen spezifischer Emulgatoreigenschaften und der Strömungsverhältnisse in Dispergierspalt. Thèse, université de Karlsruhe.

Barthès-Biesel, D. et Acrivos, A. (1973) Deformation and Burst of a Liquid Droplet Suspended in a Linear Shear Field. J. Fluid Mech., 61, 1 sq.

Basedow, A.M. et Ebert, K.H. (1977) Ultrasonic Degradation of Polymers in Solution. Adv. Polymer Sci., 22, 83 sq.

Becher, P. (1965) Chap. 7, in Emulsions: Theory and Practice, $2^{\mathrm{e}}$ éd., Reinhold, New York.

Caroll, B.J. et Lucassen, J. (1976) Chap. 1, in Theory and Practice of Emulsion Technology, Smith, A.L. (éd.), Academic, Londres.

Chandrasekhar, S. (1961) Chap. 10, 11 et 12, in Hydrodynamic and Hydromagnetic Stability, Clarendon, Oxford.

Collins, S.E. et Bowen, W.R. (1997) Membrane Emulsification, in Proc. Deuxième Congrès mondial de l'émulsion, Bordeaux, 23-26 septembre, 1, 1-2/215 sq.

Cox, R.G. (1969) The Deformation of a Drop in a General TimeDependent Fluid Flow. J. Fluid Mech., 37, 601 sq.

Davies, J.T. (1972) Chap. 8, 9 et 10, in Turbulence Phenomena, Academic Press, New York et Londres.

Davies, J.T. (1985) Drop Sizes of Emulsions Related to Turbulent Energy Dissipation Rates. Chem. Eng. Sci., 40, 839 sq.

Davies, G.A, Nilsen, F.P. et Gramme, P.E. (1996) The Formation of Stable Dispersions of Crude Oil and Produced Water: The Influence of Oil Type, Wax et Asphaltene Content, in Proc. of SPE Annual Technical Conference and Exhibition, Denver, Colorado, 6-9 octobre, 163-171.

Desnoyer, C., Masbernat, O. et Gourdon, C. (1998) Experimental Study of Drop Size Distribution at High Phase Fraction in LiquidLiquid Dispersion, in Proc. of Third International Conference on Multiphase Flow, Lyon, 8-12 juin.

Flanigan, D.A., Stolhand, J.E., Shimoda, E. et Skilbeck, F. (1992) Use of Low-Shear Pumps and Hydrocyclones for Improved Performance in the Cleanup of Low-Pressure Water. SPE Production Engineering, 7, 3, 295 sq. 
Gidley, J.L. et Hanson, H.R. (1974) Central Terminal Upset from Well Treatment is Prevented. Oil et Gas J., 72, 6, 53 sq.

Gopal, E.S.R. (1968) Chap. 1, in Emulsion Science, Sherman, P. (éd.), Academic, New York et Londres.

Gouda, J.H. et Joos, P. (1975) Application of Longitudinal Wave Theory to Describe Interfacial Instability. Chem. Eng. Sci., 30, $521 s q$.

Grace, H.P. (1982) Dispersion Phenomena in High Viscosity Immiscible Fluid Systems and Application of Static Mixers as Dispersion Devices in such Systems. Chem. Eng. Commun., 14, $225 s q$.

Hinze, J.O. (1955) Fundamentals of the Hydrodynamic Mechanism of Splitting in Dispersion Processes. Am. Inst. Chem. Eng. J., 1,289 sq.

Janssen, J.J.M., Boon, A. et Agterof, W.G.M. (1994) Influence of Dynamic Interfacial Properties on Droplet Breakup in Simple Shear Flow. Am. Inst. Chem. Eng. J., 40,1929 sq.

Karabelas, A.J. (1978) Droplet Size Spectra Generated in Turbulent Pipe Flow of Dilute Liquid/Liquid Dispersions. AIChE Journal, 24, 2, 170 sq.

Karbstein, H. et Schubert, H. (1995) Developments in the Continuous Mechanical Production of Oil-in-Water MacroEmulsions. Chem. Eng. and Proc., 34,205 sq.

Kiefer, P. (1977) Thèse, université de Karlsruhe.

Kumar, R. et Saradhy, Y.P. (1972) Ind. Eng. Chem. Fund., 11, $307 s q$.

Kumar, S., Kumar, R. et Ghandi, K.S. (1991) Alternative Mechanisms of Drop Breakage in Stirred Vessels. Chem. Eng. Sci., 46, 10, 2483 sq.

Kurzhals, H.A. (1977) Thèse, université de Hanovre.

Lagisetty, J.S., Das, P.K., Kumar, R. et Ghandi, K.S. (1986) Breakage of Viscous and Non-Newtonian Drops in Stirred Dispersions. Chem. Eng. Sci., 41, 1, 65 sq.

Levich, V.G. (1962) Chap. 5 et 8, in Physicochemical Hydrodynamics, Prentice-Hall, Englewood Cliffs.

Lucassen-Reynders, E.H. and Lucassen, J. (1969) Properties of Capillary Waves. Adv. Colloid Interface Sci., 2, 347 sq.

Lucassen-Reynders, E.H. et Kuijpers, K.A. (1992) The Role of Interfacial Properties in Emulsification. Colloids and Surfaces, 65, $175 s q$.

Lucassen, J. (1979) Physical Chemistry of Surfactant Action, Lucassen-Reynders, E.H. (éd.). Surfactant Sci. Series, 10, Marcel Dekker, New York.

Manning, F.S. et Thompson, R.E. (1995) Chap. 4, in Water-inCrude Oil Emulsions. Oilfield Processing, Vol. Two: Crude Oil, 39 sq.

Mason, T.G. et Bibette, J. (1996) Emulsification in Viscoelastic Media. Physical Review Letters, 77, $3481 \mathrm{sq.}$

Mason, T.G. et Bibette, J. (1997) Shear Rupturing of Droplets in Complex Fluids, Langmuir, 13, $4600 \mathrm{sq}$.

Mikami, T., Cox, R.G. et Mason, S.G. (1975) Breakup of Extending Liquid Threads. Int. J. Multiphase Flow, 2, 113 sq.

Percy, J.S. et Sleicher, C.A. (1983) Drop Breakup in the Flow of Immiscible Liquids Through an Orifice in a Pipe. AIChE Journal, 29, 1, $161 s q$.

Richardson, E.G. (1953) Chap. 2, in Flow Properties of Disperse Systems, Hermans, J.J. (éd.), North-Holland, Amsterdam.

Ronningsen, H.P. et Urdahl, O. (1995) A North Sea Crude Oil and its Water-in-Crude Oil Emulsions. Comparison between Small Scale Laboratory Experiments and More Realistic Conditions, in Proc. of 7th BHR Group LTD et al. Multiphase
Prod. Int. Conf., Cannes, 7-9 juin. BHR Group Conf. Ser. Publication, 14, 33 sq.

Rumscheidt, F.D. et Mason, S.G. (1962)J. Colloid Sci., 17, 260 sq.

Schramm, L.L. (1992) Petroleum Emulsions: Basic Principles, in Emulsions: Fundamentals and Applications in the Petroleum Industry, Schramm, L.L. (éd.). ACS Advances in Chemistry Series, 231, 1 sq.

Schubert, H. et Ambruster, H. (1989) Prinzipien der Herstellung und Stabilität von Emulsionen. Chem.-Ing.-Tech., 61, 701 sq.

Schubert, H. et Stang, M. (1997) New Developments in the Production of Food Emulsions, in Proc. Deuxième Congrès mondial de l'émulsion, Bordeaux, 23-26 septembre, 4, 327 sq.

Shea, G.B. (1939) Practices and Methods of Preventing and Treating Crude-Oil Emulsions. Bulletin 417, US Bureau of Mines, Washington, DC.

Sleicher, C.A. (1962) Maximum Stable Drop Size in Turbulent Flow. AIChE Journal, 8, 4, 471 sq.

Spielman, L.A. (1978) The Scientific Basis of Flocculation, Ives, K.J. (éd.), Sijthoff et Noordhoff, Alphen a. d. Rijn, 63 sq.

Stang, M., Karbstein, H. et Schubert, H. (1994) Adsorption Kinetics of Emulsifiers at Oil-Water Interfaces and their Effect on Mechanical Emulsification. Chem. Eng. Proc., 33, 307 sq.

Stang, M., Maze, H. et Schubert, H. (1997) Influence of Continuous Phase Viscosity on Droplet Size in Continuous Emulsification, in Proc. Deuxième Congrès mondial de l'émulsion, Bordeaux, 23-26 septembre, 1, 1-2/289 sq.

Stone, H.A. (1994) Dynamics of Drop Deformation and Breakup in Viscous Fluids. Annu. Rev. Fluid Mech., 26, 65 sq.

Svetgoff, J.A. (1989) Demulsification Key to Production Efficiency. Petroleum Engineer International, 61, 8, $28 \mathrm{sq.}$

Taisne, L., Walstra, P. et Cabane, B. (1996) Transfer of Oil between Emulsion Droplets. Journal of Colloid and Interface Science, 184, 378 sq.

Taisne, L., Walstra, P. et Cabane, B. (1997) Recoalescence during Emulsification, in Proc. Deuxième Congrès mondial de l'émulsion, Bordeaux, 23-26 septembre, 1, 1-2/014 sq.

Taisne, L. (1997) Échanges d'huile entre gouttes d'émulsion. Thèse, université Paris 6.

Taylor, G.I. (1934) Proc. Royal Soc., A146, 501 sq.

Van der Zande, M.J. et van den Broek, W.M.G.T. (1998) Breakup of Oil Droplets in the Production System, in Proc. of ASME Energy Sources Technology Conference and Exhibition, Houston, 2-4 février. ETCE98-4744.

Walstra, P. (1974) Influence of Rheological Properties of Both Phases on Droplet Size of O/W Emulsions Obtained by Homogeneization and Similar Processes. Dechema Monogr., 77, 87 sq.

Walstra, P. (1983) Formation of Emulsions, in Encyclopedia of Emulsion Technology, vol. 1, Becher, P. (éd.), Marcel Dekker, New York.

Walstra, P. (1993) Principles of Emulsion Formation. Chem. Eng. Sci., 48,333 sq.

Walstra, P. (1997) Formation of Emulsions, in Proc. Deuxième Congrès mondial de l'émulsion, Bordeaux, 23-26 septembre, 4, $67 s q$.

Yaws, C.L., Yang, H.C., Hopper, J.R. et Hansen, K.R. (1990) Hydrocarbons: Water Solubility Data. Chemical Engineering, 97, 4, $177 \mathrm{sq}$.

Zana, E. et Leal, L.G. (1974) Proc. Intern. Colloquium on Drops and Bubbles, Collins, D.J., Plesset, M.S. et Saffren, M.M. (éd.), Jet Propulsion Laboratory, Pasadena, Californie, 428 sq.

Manuscrit définitif reçu en décembre 1999 